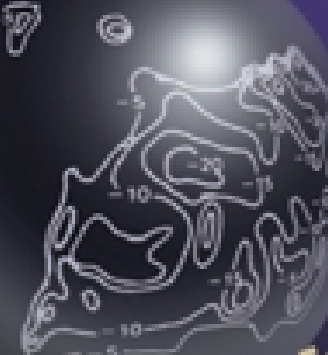

Live Seience and Tocolys 50 oth Anniversary

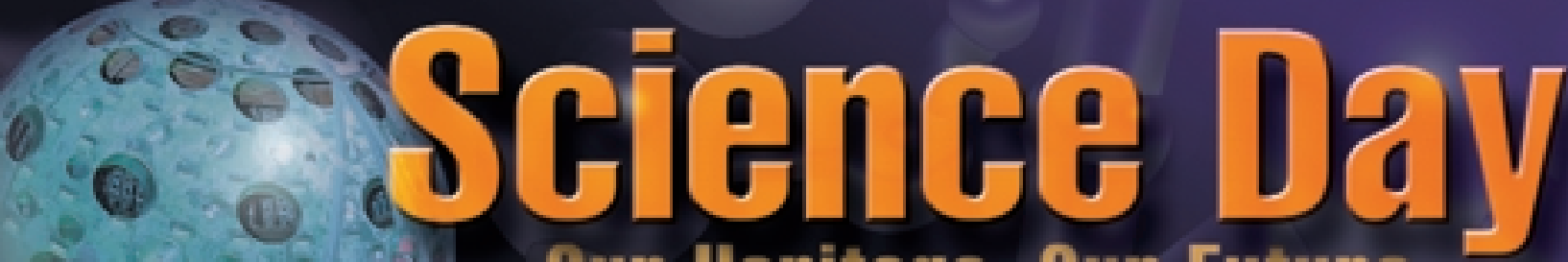

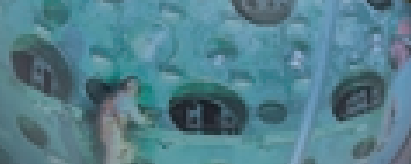

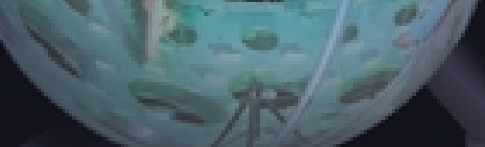

\title{
Our Heritage, Our Future
}
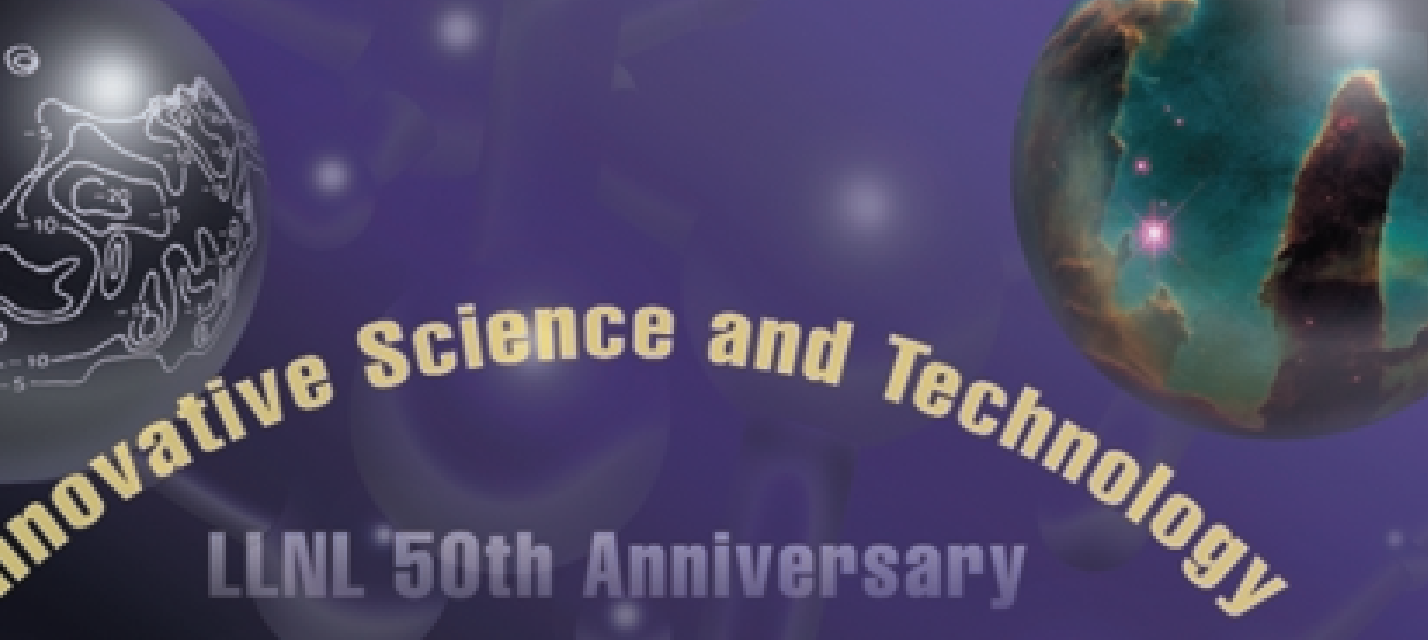


\section{DISCLAIMER}

This document was prepared as an account of work sponsored by an agency of the United States Government. Neither the United States Government nor the University of California nor any of their employees, makes any warranty, express or implied, or assumes any legal liability or responsibility for the accuracy, completeness, or usefulness of any information, apparatus, product, or process disclosed, or represents that its use would not infringe privately owned rights. Reference herein to any specific commercial product, process, or service by trade name, trademark, manufacturer, or otherwise, does not necessarily constitute or imply its endorsement, recommendation, or favoring by the United States Government or the University of California. The views and opinions of authors expressed herein do not necessarily state or reflect those of the United States Government or the University of California, and shall not be used for advertising or product endorsement purposes.

This work was performed under the auspices of the U. S. Department of Energy by the University of California, Lawrence Livermore National Laboratory under Contract No. W-7405-Eng-48.

This report has been reproduced directly from the best available copy.

Available electronically at http://www.doc.gov/bridge

Available for a processing fee to U.S. Department of Energy

And its contractors in paper from

U.S. Department of Energy

Office of Scientific and Technical Information

P.O. Box 62

Oak Ridge, TN 37831-0062

Telephone: (865) 576-8401

Facsimile: (865) 576-5728

E-mail: reports@adonis.osti.gov

Available for the sale to the public from

U.S. Department of Commerce

National Technical Information Service

5285 Port Royal Road

Springfield, VA 22161

Telephone: (800) 553-6847

Facsimile: (703) 605-6900

E-mail: orders@ntis.fedworld.gov

Online ordering: http://www.ntis.gov/ordering.htm

OR

Lawrence Livermore National Laboratory

Technical Information Department's Digital Library

http:/ / www.llnl.gov/tid/Library.html 


\section{Lawrence Livermore National Laboratory}

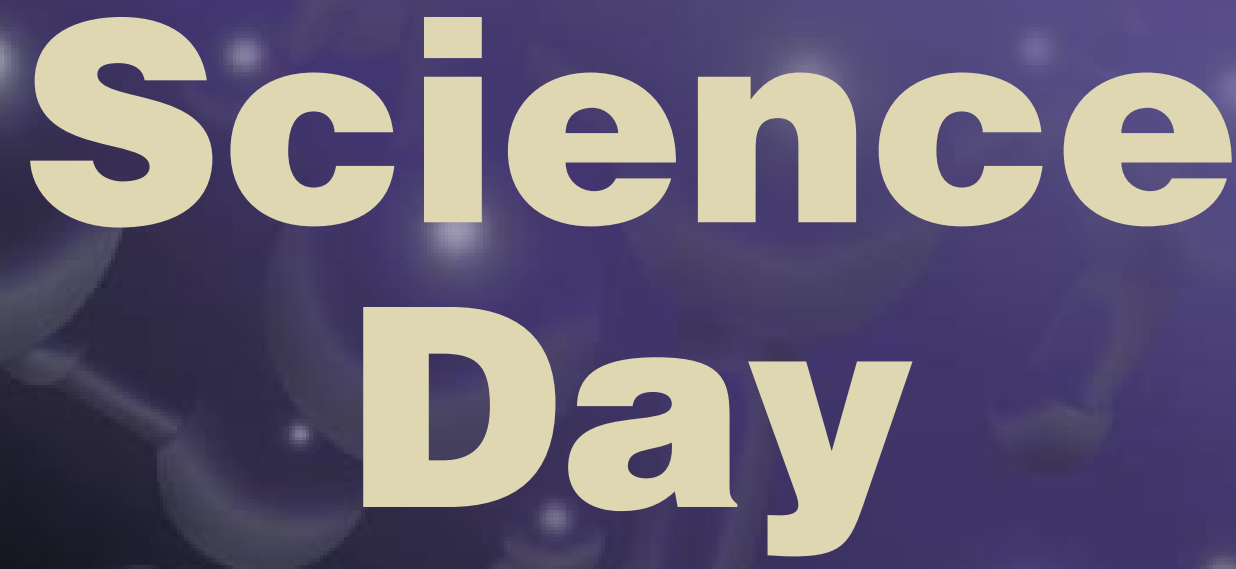

\section{Abstracts}

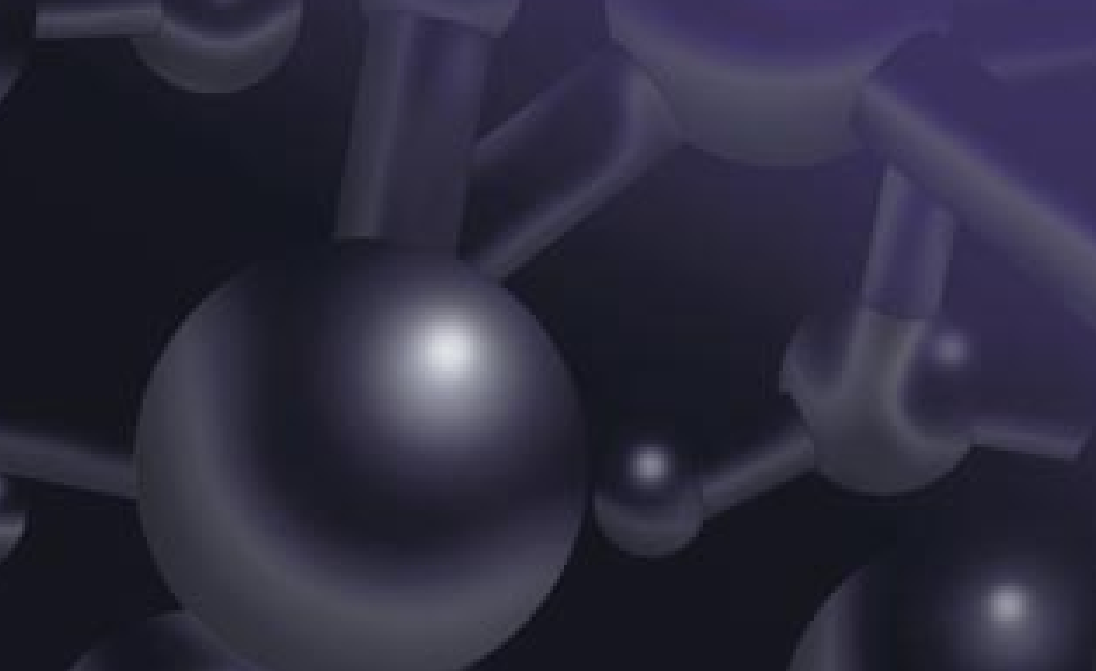

September 17, 2002

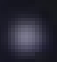

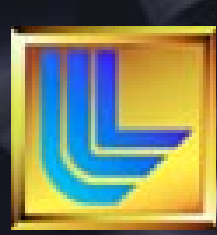

Laboratory Science and Technology Office

P.O. Box 808, L-3

Livermere, CA 94551

Karen Kline, Technical Editor Amy Henke, Graphic Design

Cover-Daniel Moore

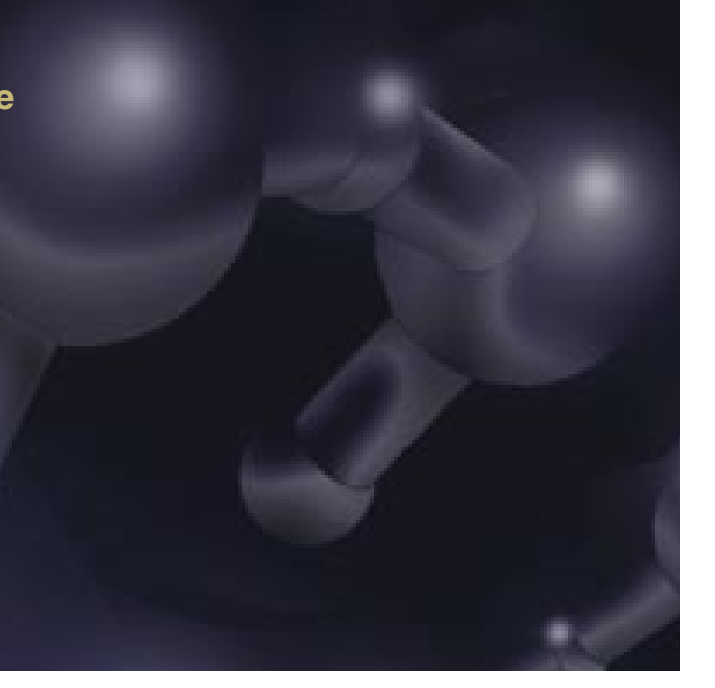


Invited Presentations

Abstracts

\section{Biological Research Session}

Session Overview

LLNL Bioscience History -Contributions on a Rapidly Unfolding Canvas

Bert Weinstein, Session Chair

Some Early History of Biological Research at LLNL

Mort Mendelsohn

Cytometry to Cancer Genomics: A Meandering Walk from Physics to Cancer Genetics

Joe Gray

The Role of Detection in Biodefense

Fred Milanovich

The Book of Life from Anthrax to Zebra: Biology beyond the DNA-Sequencing Era

Lisa Stubbs

\section{Climate Modeling Session}

Session Overview

Climate, Carbon, and Computing at Livermore: From Nuclear Winter to Global Warming, and beyond Bill Dannevik, Session Chair

Early Atmospheric Model Development at LLNL

Chuck Leith

The Origins of Climate and Chemistry Modeling at LLNL: Making a Difference Globally

Mike MacCracken

Understanding Past and Future Climate Changes: LLNL's Recent Contributions

Phil Duffy

\section{Astrophysics Session}

Session Overview

Astrophysics at Livermore

Bruce Tarter, Session Chair

Explosions at Livermore, or Explosions I Have Known and Don't Understand, including Supernovae Stirling Colgate

Highlights of the Seventies and Eighties

Bruce Tarter 
Adaptive Optics: A Sharper View of the Universe

Claire Max

The True Heart of a Star

Dave Dearborn

\section{Fusion Session}

Session Overview

Inertial Fusion and High-Energy-Density Research at LLNL

George Miller, Session Chair

Early Steps towards Inertial Confinement Fusion

John Nuckolls

High-Energy-Density Physics, with Applications to Astrophysics

Bruce Remington

Development of the Scientific Foundation of Inertial Confinement Fusion John Lindl

Inertial Fusion and High-Energy-Density Science on the National Ignition Facility

George Miller

\section{Presenters}

\section{Poster Expo}

Abstracts

Active Interrogation of Cargo Containers

Arden Dougan et al.

Adaptive Optics in Astronomy and on Earth

Bruce Macintosh et al.

Advances in High-Performance Computing at LLNL

Bing Young

Applications of Ion-Beam Analytical Techniques to Environmental and Biomedical Research at the Center for Accelerator Mass Spectrometry

John Knezovich et al.

Applied Structural Genomics of Human and Microbial Proteins

Matthew A. Coleman, Peter T. Beernink, and Brent W. Segelke 
Autonomous Pathogen Detection System (APDS) Offers Rapid and Continuous Monitoring of Multiple Airborne Biological Threat Agents

Steve Brown et al.

Biological and Materials Science Applications of Single-Molecule Spectroscopy

Chris Hollars et al.

Biophotonics for Medicine

Duncan Maitland et al.

BlueGene/L-The Next Generation of Scalable Computing Platform

Mark Seager, Lynn Kissel, and Kim Yates

Computational Characterization of Biomolecules

Daniel Barsky et al.

Continuum Solvent Model for First-Principles Molecular Dynamics Simulations

Jean-Luc Fattebert and Francois Gygi

Critical Skills Internships - Science \& Technology Education Program

Don Correll and Barry Goldman

Development of Synthetic High-Affinity Ligands

Julie Perkins

Distribution of Spore Particles and Decontamination Agents in an Office Environment

Paula Krauter et al.

DNA Compaction Induced by the Yeast Mitochondrial Protein ABF2p

Larry Brewer et al.

Effects of Trichloroethylene (TCE) on the Mobilization of Metals in Livermore

Richard A. Brown

Electromagnetic Imaging of $\mathrm{CO}_{2}$ Sequestration at an Enhanced-Oil-Recovery Site

Barry Kirkendall and Jeff Roberts

Evaluation of Flor-Essence ${ }^{\circledR}$ and Essiac ${ }^{\circledR}$ Herbal Tonics using In Vitro and In Vivo

Models for Breast Cancer Research

L. Michelle Bennett and Kristen Kulp

Experimental and Theoretical Considerations for Modeling Material Performance in a Nuclear Waste Repository

Tammy Summers et al.

Experiments to Develop an Energetic, High-Intensity Laser-Based Proton Source Pravesh Patel 
A Geospatial Model for Remedial Design Optimization and Performance Evaluation

V. Madrid

Industrial Partnering with Lawrence Livermore National Laboratory

Catherine Elizondo

Information Security in a Hacker's World

John Allen et al.

Institutional Publications

Technical Information Department Team

Interactive Visualization of Terabyte-Sized Datasets

Valerio Pascucci

Laboratory Simulation of the X-Ray Emission from Comets

Peter Beiersdorfer

Laser-Plasma Interaction Experiments on Nonlinear Saturation of Stimulated Brillouin and Raman Scattering

Siegfried Glenzer et al.

Liquid-Bead-Based Biological Detection Assays

Fred Milanovich

Making Climate Models Better using Weather Forecasts and Observations

Gerald Potter et al.

Making Peptides in Cometary Impacts

Jennifer Blank

Mechanical Properties of Nanocrystalline Nickel-Atomic Scale Simulations and Experimental Observations

Maria J. Caturla

Mechanisms of Pathogenicity

J. Patrick Fitch and Sandra McCutchen-Maloney

Microfluidic Tools for Biological Sample Preparation

Robin Miles et al.

Molecular Dynamics Simulations of the Editing Subunit of E. coli DNA Polymerase III:

An Essential Protein in DNA Replication

Edmond Lau and Felice Lightstone

Nanoscience and Nanotechnology for Nonproliferation Applications

John Reynolds and Thomas Tillotson

The National Ignition Facility

Regina Bonanno 
Neutron Detectors for Inertial Confinement Fusion

Mike Moran et al.

OPCW Certification for the Forensic Science Center

Armando Alcaraz

Keith Carlisle, Paul Geraghty, and Sam Thompson

Quantum-Level Calculations

Giulia Galli et al.

Remote Optical Characterization Sensor Suite

Joel Bowers

Removing Nitrate and Perchlorate from Groundwater using the Containerized Wetland System Paula Krauter and William Daily

Ribbon Fiber Lasers - the High-Power Lasers of the Future

Jay Dawson, Zhi Liao, Raymond Beach, and Steven Payne

A Search for the Origin of Matter/Antimatter Asymmetry: The BABAR Experiment at the SLAC-LBNL-LLNL B Factory

Douglas Wright et al.

Secure Air-Optic Transport and Routing Network

Anthony Ruggiero

A Simulations and Experimental Program to Assess Ballistic Missile Defense Lethality

Douglas Faux et al.

Solid-Oxide Fuel Cell Development at LLNL

Quoc Pham et al.

Solid-State Heat Capacity Lasers, a Compact Missile-Defense System

Mark Rotter

The Spheromak Path to Economic Fusion Energy

Simon Woodruff

Stretchable Micro-Electrode Array

Peter Krulevitch and Mariam Maghribi

Surface Passivation Effects of Deposited Germanium Nanocrystal Films Probed with

Synchrotron Radiation

Christoph Bostedt et al.

Thermodynamics and Structure of Plutonium Alloys

Patrick Allen et al. 
The True Heart of a Star

David Dearborn

Understanding Global Ozone and Aerosol Pollution using the LLNL IMPACT Chemistry Model Doug Rotman et al.

Using Human Urinary PhIP Metabolites to Study Individual Variation in Carcinogen Metabolism and Chemoprevention through Dietary Interactions

Kristen Kulp, Mark Knize, and James Felton

Views of the Solar System using Adaptive Optics at the Keck

Observatory

Seran Gibbard

What's New in University Relations

Physical Biosciences Institute

UC Merced

Laura Gilliom

The World's Largest Linux Cluster for LLNL Scientific Simulations Lynn Kissel and Mark Seager 


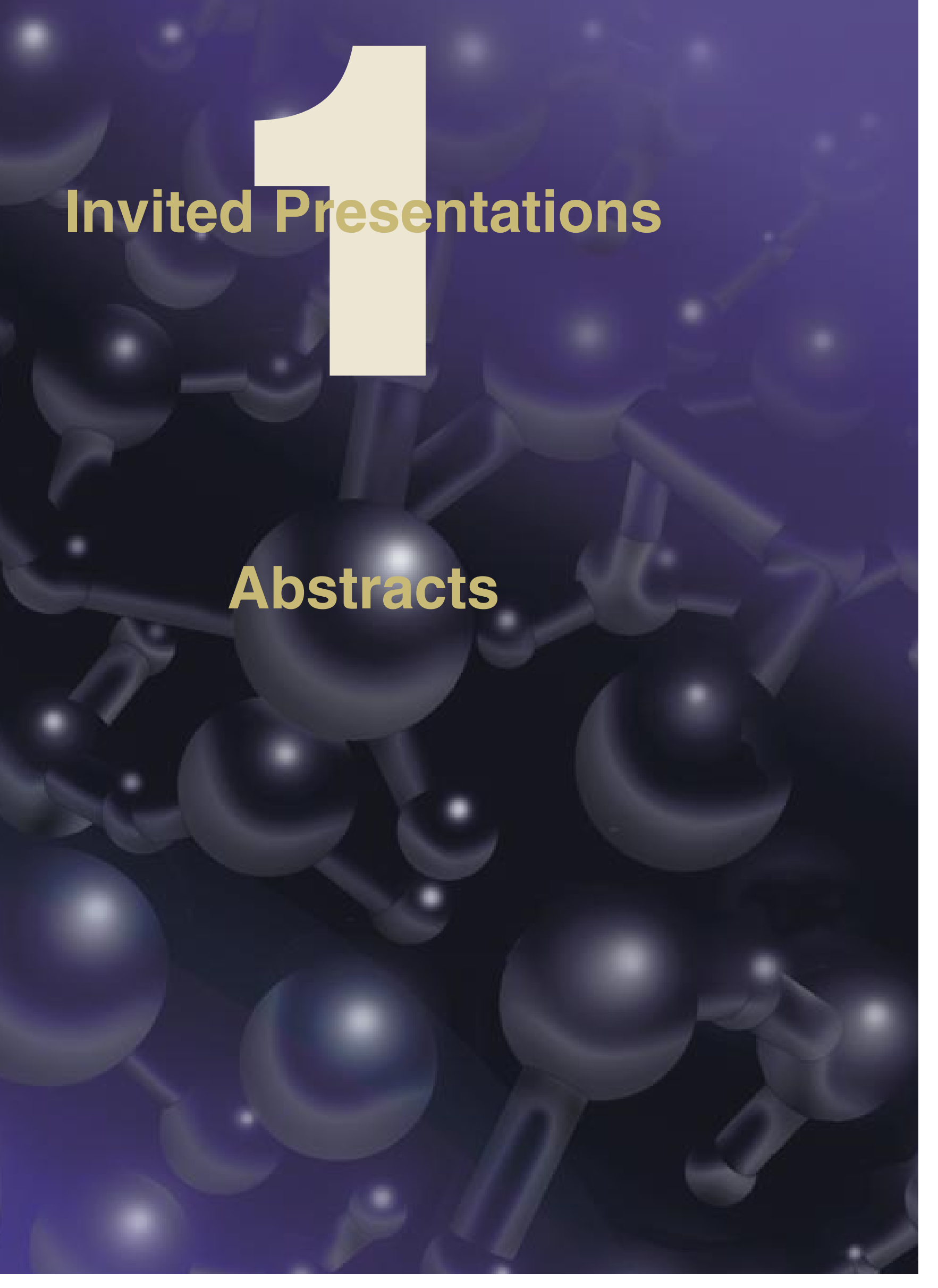




\section{LLNL Bioscience History-Contributions on a Rapidly Unfolding Canvas}



\section{Bert Weinstein}

Acting Associate Director,

Biology and Biotechnology Research Program
LLNL's achievements in bioscience are best understood against the backdrop of the revolutionary changes occurring in the field overall. In 1953, the year after LLNL was founded, Watson and Crick determined the helical structure and basepairing of DNA, revealing the chemical basis of the genetic code used by every known form of life. This discovery is to genetics roughly what Mendeleev's discovery of the periodic nature of chemical properties is to chemistry, providing a fundamental insight on which essentially all future understanding is built.

In 1963, the bioscience research program was established at LLNL as part of the Atomic Energy Commission's efforts to understand the effects of ionizing radiation. The following year, 1964, the three-letter code and chemical mechanism by which DNA directs the construction of proteins was determined.

By the mid-1970s, as LLNL's laser program was building and LLNL's bioscience program was developing methods to sort cells and chromosomes, the Sanger sequencing method was worked out, opening the door to reading modest stretches of DNA sequence.

In the 1980s, as LLNL was deeply involved in ballistic missile defense and the LLNL biology program was developing chromosome-specific libraries and chromosome painting, the polymerase chain reaction (PCR) method for amplifying sections of DNA was developed. PCR as a tool in biology holds a place akin to the invention of the transistor in physical sciences - enabling a host of previously unthinkable applications and tools.

In the 1990s, as LLNL developed the Stockpile Stewardship Program and began a major effort in nonproliferation and counterterrorism, LLNL's role in the human genome sequencing project was in full swing and the initial developments in rapid PCR and pathogen detection were laying the groundwork for our role in biodefense research.

In 1995, the first DNA sequence of an entire organism was published, the bacterium Haemophilus influenzae. In 1998, four short years ago, the first multicellular animal, the worm C. elegans, was sequenced. In the same year, the fledgling Joint Genome Institute (JGI) set and then met what seemed at the time to be an almost ridiculous goal of drafting 20 million DNA bases in a year. Today that represents less than a week of JGI production.

On Science Day, all four of our speakers have contributed to this dizzying history. Dr. Mortimer Mendelsohn, the second director of Biological Research at LLNL, provides insights into some of the founding science and personalities of LLNL's biology program. Dr. Joe Gray, Professor of Laboratory Medicine at the University of California, San Francisco, delves a bit into the history of chromosome sorting and painting. Dr. Fred Milanovich tells us about how we got started and what we have achieved in biodefense research. And finally, Dr. Lisa Stubbs describes some results of the explosion of genomic data and tools, and provides a look ahead to just a few of the possible future impacts. 


\section{Some Early History of Biological Research at LLNL}

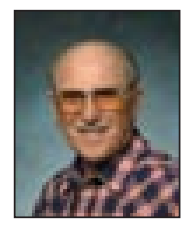

Mort Mendelsohn

Senior Scientist and former

Associate Director,

Biology and Biotechnology Research Program

LLNL was 11 years old before it inaugurated its first Biological and Environmental Research (BER) Program. Jack Gofman, a Professor at UC Berkeley, organized that Program and led it until 1972, when he handed it over to me. The initial theme was "Dose to Man," motivated by the potentially heavy consequences of Plowshare, with its use of nuclear explosives for civil engineering projects. Plowshare never got past the talking stage, but fortunately the new BER Program went ahead anyway.

Gofman, an M.D./Ph.D., was deeply into the molecular biology of cardiovascular disease, and was also one of Glenn Seaborg's graduate students during the days when plutonium was being discovered. He recruited a fresh group of biological scientists, including division leader Bernie Shore and wife Virgie, who were expert in the mechanisms of atherosclerosis; Fred Hatch, also an M.D./Ph.D. and expert in nucleic acids; Bob Taylor, a Berkeley biochemist; and Lowry Dobson, a reproductive and radiation biologist, all of whom eventually finished out their highly successful careers here at LLNL

It was Gofman himself who began the theme we are to follow today, a path that starts at the chromosome, leads to the human genome, and diverges out to genomics in general, including our own highly specialized genomic and technologic approaches to coping with bioterrorism. In the decade or two before oncogenes dominated cancer research, Gofman was searching for insights into the cancer process. Sensing that chromosomes played an important role, he wanted to examine the chromosomes of a few dozen readily available human cancer cell lines looking for common denominators. To do this, he needed an objective, quantitative, and sensitive yardstick. He chose

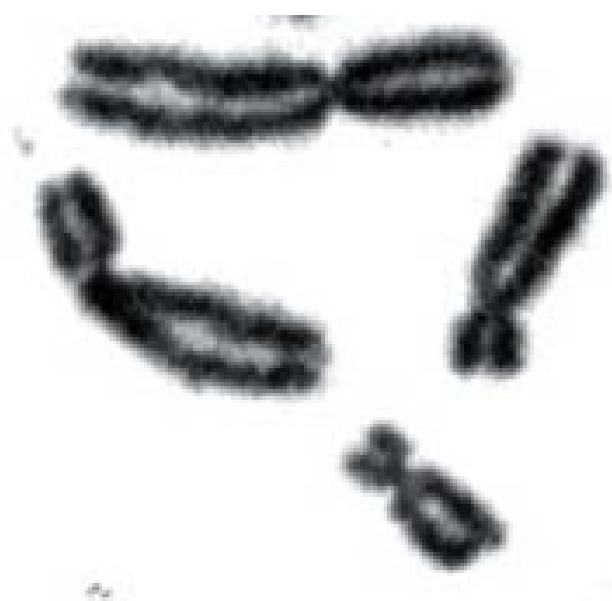

Several human chromosomes as imaged digitally (1975).

chromosome length as measured in digital images of the dividing cells.

By the early 1970s, he had built the system and made preliminary measurements. But the resolution of chromosome length was poor, and Gofman's emphasis on length measurements to the exclusion of conventional cytogenetics had alienated the rest of the chromosome community. He passed the problem and the Program over to me in 1972.

I was at the University of Pennsylvania, along with Brian Mayall, Bart Gledhill, Jan Gogel, Dan Moore, and several other future LLNL employees. We also had a digital microscope system, but in addition to our quarter-micron pixel size, we recorded 256 levels of gray and thus were able to measure optical density. We applied the system to DNA-stained human chromosomes by adding up the hundreds of absorption measurements that constituted each chromosomal image. From the resulting sums, we could directly estimate the fraction of cellular DNA attributable to each chromosome or chromosome pair. With a few years of experience, we showed that chromosomes had the same invariance of DNA content as cells, thus making DNA content a much more reliable chromosomal property than length.

With this kind of technology and with goals such as finding ways to measure the somatic mutation rate in human subjects, my Pennsylvainia group belonged at LLNL. And to round out the emphasis on cytometry, we added Marv Van Dilla, who had spearheaded the development of flow cytometry at Los Alamos. In our first year together, Marv and I hired a recent physics Ph.D. who wanted to switch from accelerators to biophysics. His name was Joe Gray, and he is giving the next talk today. 


\section{Cytometry to Cancer Genomics-A Meandering Walk from Physics to Cancer Genetics}

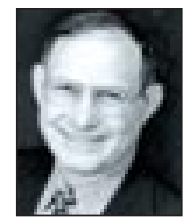

\section{Joe Gray}

Professor of Laboratory Medicine and Radiation Oncology,

University of California, San Francisco

I was recruited to the Biomedical Sciences Division of LLNL in 1972 by Drs. Marvin Van Della and Mortimer Mendelsohn to participate in the development of the then new field of flow cytometry. My first assignment was the construction of a cell sorter. This accomplished, we set out to explore possible biological uses of this new type of instrumentation. At the urging of Mort Mendelsohn, we asked whether it could be used to facilitate chromosome research. A borrowed tube of isolated chromosomes from a graduate student at UC Berkeley allowed us to discover quickly that chromosomes could be resolved and purified to an unprecedented degree with our new instruments.

The demand from the scientific community for DNA from purified chromosomes was too large to satisfy, so to meet that need, LLNL and Los Alamos established the National Laboratory Gene Library Project to construct recombinant DNA libraries containing fragments of each human chromosome type. These libraries stimulated research in two directions. First, they provided convenient
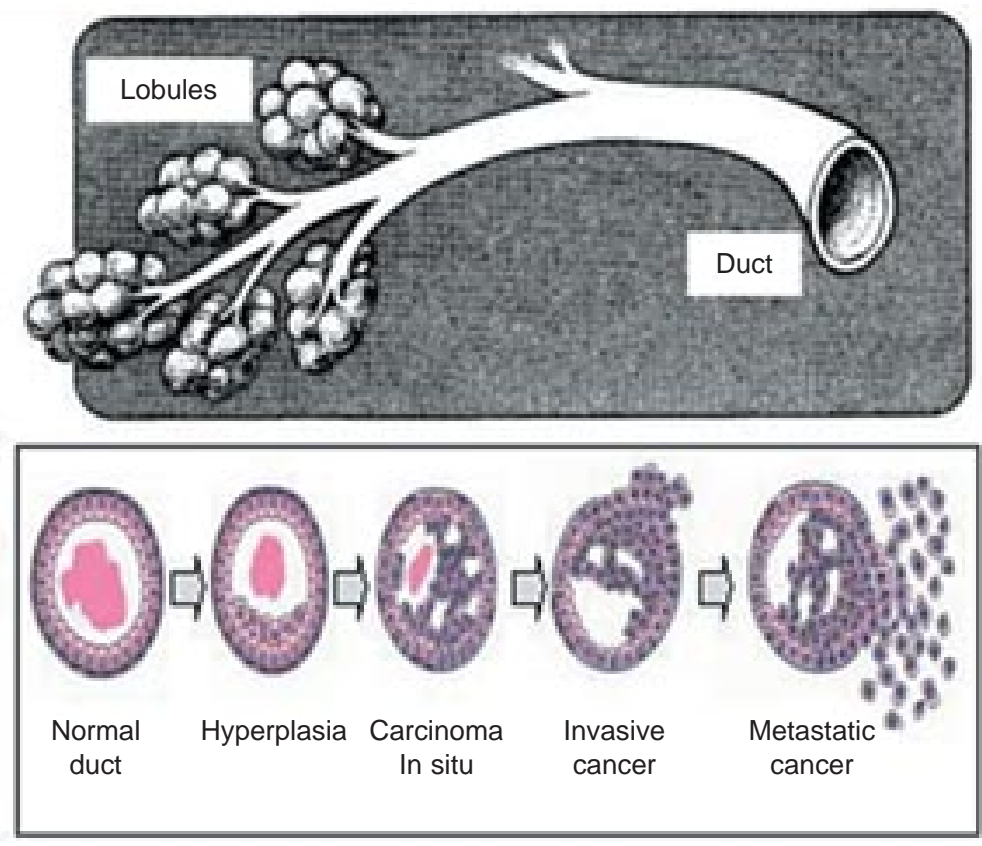

chromosome fragments that could be used for physical map construction and DNA sequencing. Construction and manipulation of these libraries demonstrated that genetic manipulation of entire chromosomes was experimentally tractable and contributed to the development of the idea that the entire genome could be mapped and sequenced. Second, they provided chromosome-specific DNA sequence collections that Dan Pinkel and I eventually used as chromosome-staining reagents using fluorescence in situ hybridization (FISH). FISH with these probes allowed specific segments of DNA in cells and chromosomes to be stained with stunning clarity and specificity, which proved to be remarkably useful in a broad range of biological and clinical applications, especially in prenatal and cancer diagnosis.

Wishing to explore the medical applications of this new technology, Dan Pinkel and I, along with several others, moved to UC San Francisco in 1991. Shortly thereafter, we developed a variation of FISH that allowed genomic aberrations in entire genomes to be mapped in a single experiment. This proved particularly useful in identifying genomic aberrations that play important roles in solid-tumor initiation and development. Using these techniques, we have now identified more than 30 regions of recurrent genomic abnormality in breast cancer, and more are being found in other solid-tumor types. The completion of the human genome is facilitating discovery of the genes in these regions that contribute to the cancer phenotype. Our work now focuses on how this information can be used to facilitate earlier cancer detection, to better understand mechanisms of tumor formation, to improve prediction of individual response to therapy, and to identify novel targets for gene-specific cancer therapy.

Breast cancers typically originate in the epithelial cells that line the milk ducts. Cancers are thought to arise as a result of genetic and epigenetic events that increase proliferation and reduce cell death (hyperplasia). Additional events enable the abnormal cells to fill and traverse along the ducts (carcinoma in situ), and eventually to escape from the ducts (invasive cancer) and then from the breast itself (metastatic cancer). 


\section{The Role of Detection in Biodefense}

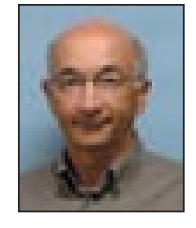

\section{Fred Milanovich}

Director Emeritus, Chemical/Biological National Security Program, R Division, Nonproliferation, Arms Control, and International Security Directorate

By the mid-1990s, the U.S. Congress began to assess the vulnerability of the U.S. civilian population to biological terrorism and found us considerably lacking in our ability to cope with even a small-scale biological event. Initially, it was thought that existing Department of Defense technology could be readily transferred to the civilian arena. But further study revealed that although military and civilian defense needs overlap, marked differences exist in the biological arena. The civilian population is neither trained nor equipped, is vulnerable to any conceivable pathogen, and often gathers in large crowds (at special events, sporting venues, etc.), where a small release has the potential to infect thousands. In response to these differences, federal agencies, including the Department of Energy, began funding directed research efforts to reduce civilian biological terrorist vulnerabilities.

A bioterrorism first
responder evaluating
LLNL portable
biodetection
technology.

A bioterrorism first responder evaluating biodetection nology.
About this time, Don Prosnitz of the Nonproliferation, Arms Control and International Security (NAI) Directorate approached me about LLNL's potential to contribute to this national security need. With Don's guidance and the support of NAI associate director Wayne Shotts and the Laboratory Directed Research and Development Program (LDRD), we established the Chemical/Biological

Nonproliferation Program (CBNP), which has grown from an initial LDRD investment of $\$ 600 \mathrm{~K}$ to a nearly $\$ 40 \mathrm{M}$ per year, federally funded program, which is currently led by Pat Fitch. CBNP's rapid growth can be attributed to LLNL's broad multidisciplinary science and engineering base and a culture that embraces cross-discipline teaming. Indeed, our early achievements, in collaboration with engineer Ray Mariella, rode the coattails of an already well-established engineering and biology partnership in support of genomics.

This presentation will provide a brief overview of the biothreat, elaborate on the Laboratory's achievements in countering the threat of bioterrorism, and give some insight into the influence of infectious disease on the survivability of humankind.

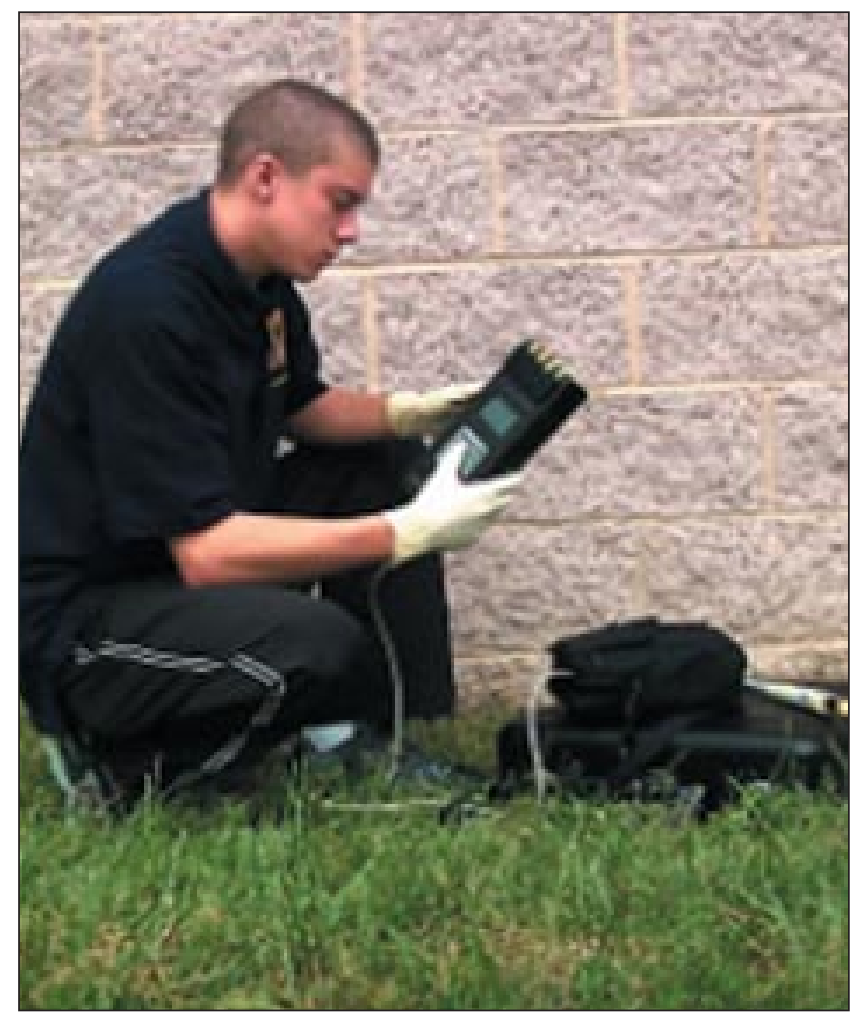




\section{The Book of Life from Anthrax to Zebra: Biology beyond the DNA Sequencing Era}

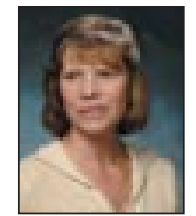

\section{Lisa Stubbs}

Acting Director, Genome Regulation and Function Division,

Biology and Biotechnology Research Program

The past several years have been an era of tremendous change for the biological sciences, starting with the race toward a goal that was deemed impossible not long ago: the complete sequence of the human genome. In the fiercely competitive drive to complete the human genome sequence, important new strides were made that have sped the reading and interpretation of the genome sequence dramatically, transforming the "impossible" to an almost routine task in a matter of several years. With these new methods, the genomes of animals-human, mouse, rat, pufferfish, the sea squirt, zebrafish, chicken, frog-and several plants, including the experimental organism, Arabidopsis, rice, and the poplar, will be completely revealed within the next two years. In addition, the genomes of countless microbes, which represent the overwhelming

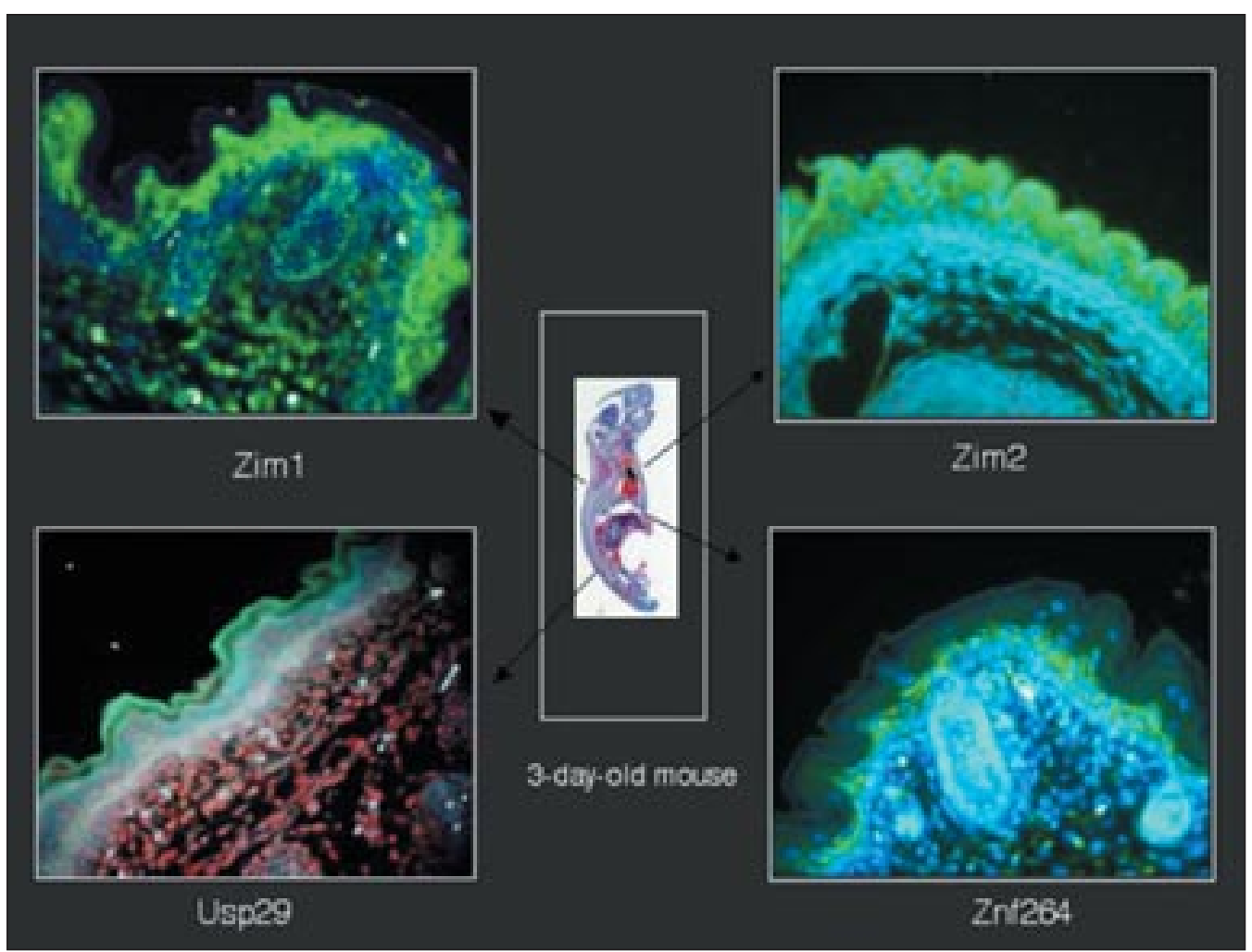

In this picture, expression of four different genes is measured in different layers of the skin in a 10$\mathrm{mm}$ section of a 3-day-old mouse. The section fits onto a microscope slide so that each of the millions of cell types in the animal's body can be examined under the microscope. In these photos, each gene's RNA product is detected by a specific probe that is tagged with a fluorescent green label to mark the sites of the gene's activity in individual cells. These experiments allow genes to be correlated with different metabolic and developmental processes, and with specific types of diseases. Since mouse and human genes are so similar, information derived from animal cells can often be extrapolated accurately to humans. 


\section{Climate, Carbon, and Computing at Livermore: From Nuclear Winter to Global Warming, and Beyond}

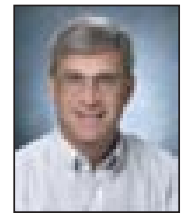

Bill Dannevik

Division Leader, Atmospheric Sciences Division, Energy and Environment Directorate

Research on the origins and impacts of global climate variations has been a mainstay of Livermore's atmospheric science applications program for five decades. The work began in the late 1950s, with the development of computer codes simulating the threedimensional global atmospheric circulation. These codes were developed, in part, to provide an unclassified application problem that would explore and stretch the limits of the emerging supercomputer industry's latest systems. Chuck Leith was a pioneer in this field; his talk describes his early adventures with the Livermore Advanced Research Computer.

Livermore's atmospheric science research then took up the question of what might happen to climate and weather as various "unnatural" materials are added to the atmosphere, whether it be dust and fallout from a thermonuclear air burst or greenhouse gases and aerosol from fossil fuel combustion. Separating the signal of such activities from the noise of natural climate variation has turned out to be a very hard problem that will challenge scientists for years to come. Mike MacCracken's talk describes the highlights and successes of this era, ranging from the late 1960s to the early 1990s, including the growth of a complementary program to predict the dispersion of hazardous materials on shorter length- and timescales.

Over the past several years, Livermore's climate-change research has centered on the detection and attribution of climate change in the observational record, on the evaluation and intercomparison of all of the world's leading climate simulation models, on the linkage of climate variations with atmospheric chemistry and aerosol physics processes, and on the application of the world's most powerful computers to the task of estimating regional-scale impacts of climate change. Phil Duffy provides an overview of this work and a glimpse of where this research may lead over the next few years.

Several themes have characterized Livermore's involvement in this research. (1) Climate change research is an increasingly multidisciplinary endeavor, requiring climate scientists to work with chemists, aerosol physicists, biologists, oceanographers, and others to sort through the multiple couplings of a wide range of processes. (2) The climate system is enormously complicated, and the corresponding simulation models require the most powerful computing systems. And, (3) the close interplay between evaluation of these models and the collection and analysis of huge quantities of experimental data pose daunting challenges in the management and mining of large scientific datasets. These enduring themes are hallmarks of many of Livermore's most successful research programs, and will continue to characterize the Laboratory's efforts for years to come. 


\title{
Early Atmospheric Model Development at LLNL
}

\author{
Chuck Leith \\ Senior Physicist, \\ A Division, \\ Defense and Nuclear Technologies Directorate
}

In the late 1950s, I had finally completed my Ph.D. research at UC Berkeley and was looking for some new direction. At that time, there was a cessation of nuclear-weapons testing, which I believed-wrongly, as it turned out-indicated an end to interest in nuclear weapons development. With the skill that I had acquired in numerical simulation of hydrodynamics and radiation transport, I thought that I might be able to contribute to the development of numerical simulation of the atmosphere.

In the fall of 1960, LLNL was acquiring a new computer, the Livermore Advanced Research Computer (LARC), which was ten times more powerful than existing computers. I visited Jule Charney and Norman Phillips at MIT, who encouraged me to go ahead.

I learned something about the equations of atmospheric dynamics from a paper by Arnt Eliason, and spent the summer of 1960 on a visit to the late
Rossby's institute in Stockholm, putting together a five-level hemispherical atmospheric model using a $5 \times 5$-degree horizontal mesh. I was sufficiently naïve to include water vapor in a hydrological cycle, which had frightened away other early modelers such as Mintz at UCLA and Smagorinsky at GFDL. In the institute library, I found a copy of the classic text on the subject by L. F. Richardson (which has since been reprinted by Dover in a paperback edition).

To display the results of the model runs, I generated contour maps of various fields such as the 500-millibar geopotential height field on an oscilloscope, which I then photographed with a 35-millimeter camera. The resulting strips of black and white film were then merged in different colors by Pacific Title Company in Hollywood to make the color film strips to be displayed.

I wish to thank the LLNL directors at the time, Edward Teller and Harold Brown, for their encouragement and support.

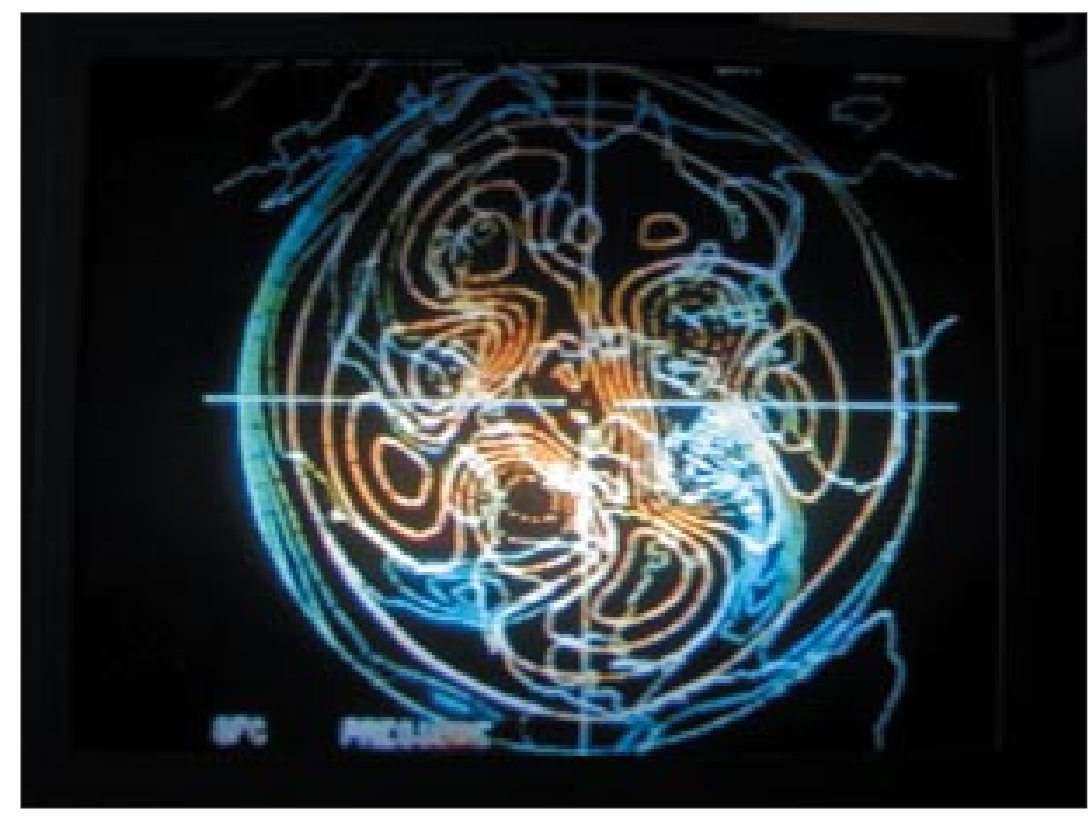

Model weather maps of the Northern Hemisphere (Polar projection) showing surface pressure (yellow) and precipitation (blue), created by the Livermore Advanced Research Computer (LARC) in 1963. Continent outlines are shown in white. 


\section{Climate Modeling at Livermore-Making a Difference Globally}

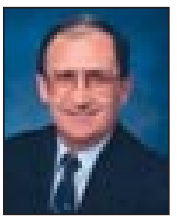

\author{
Mike MacCracken \\ Senior Global Change Scientist, \\ Office of the U. S. Global Change \\ Research Program \\ (on assignment from LLNL)
}

With the growing public interest in environmental protection in the late 1960s, two areas of research provided the basis for the development and growth of the Laboratory's atmospheric and geophysical sciences research program. One tap root was the growing capability to simulate complex systems with computers, as evidenced by Leith's development of the first global atmospheric generalcirculation model. The second tap root was the intensifying need to know what happened and would happen when radionuclides were injected into the atmosphere. The combination of these two efforts led Laboratory researchers to pursue a wide array of research activities that examined real and potential impacts that could affect the climate and environmental pollution. For example, climate modeling research studies examined the potential effects of a nuclear war scenario, including a fleet of supersonic aircraft, deforestation, volcanic aerosols, increased concentrations of $\mathrm{CO}_{2}$ and other greenhouse gases, and lofting of dust and smoke. To test the validity of such simulations, diagnostic and intercomparison studies delved into the basis for feedbacks and uncertainties in radiation and climate models. Environmental pollution studies considered, for example, the causes of smog in the Bay Area; the effects of nuclear testing, supersonic aircraft, and chlorofluorocarbons (i.e., freons) on the stratospheric ozone layer and UV radiation at the surface; and the transport and deposition of radionuclides and other substances emitted from a variety of sources, ranging from the Three Mile Island and Chernobyl reactor accidents to the Gulf War. In several of these instances, the efforts that began here ended up being incorporated into national and international policies. Research now is moving more and more toward studying both climate and environmental pollution in a coupled mode.
In 1983, based on very simple model simulations, Carl Sagan and others proposed that the smoke lofted from fires started by a nuclear war would cause the entire planet to fall into a "nuclear winter." Much more realistic studies done at Livermore made clear that while there would be cooling over land areas, the world would not freeze and that, as before this suggestion, the massive destruction resulting from a nuclear exchange was appropriately the major deterrent. This figure shows the cooling calculated to result a few weeks after a full strategic nuclear exchange that burned all major cities and led to the lofting of 150 billion tons of soot. The results show midcontinental summertime temperatures dropping to near freezing, with most areas suffering much milder climatic effects.

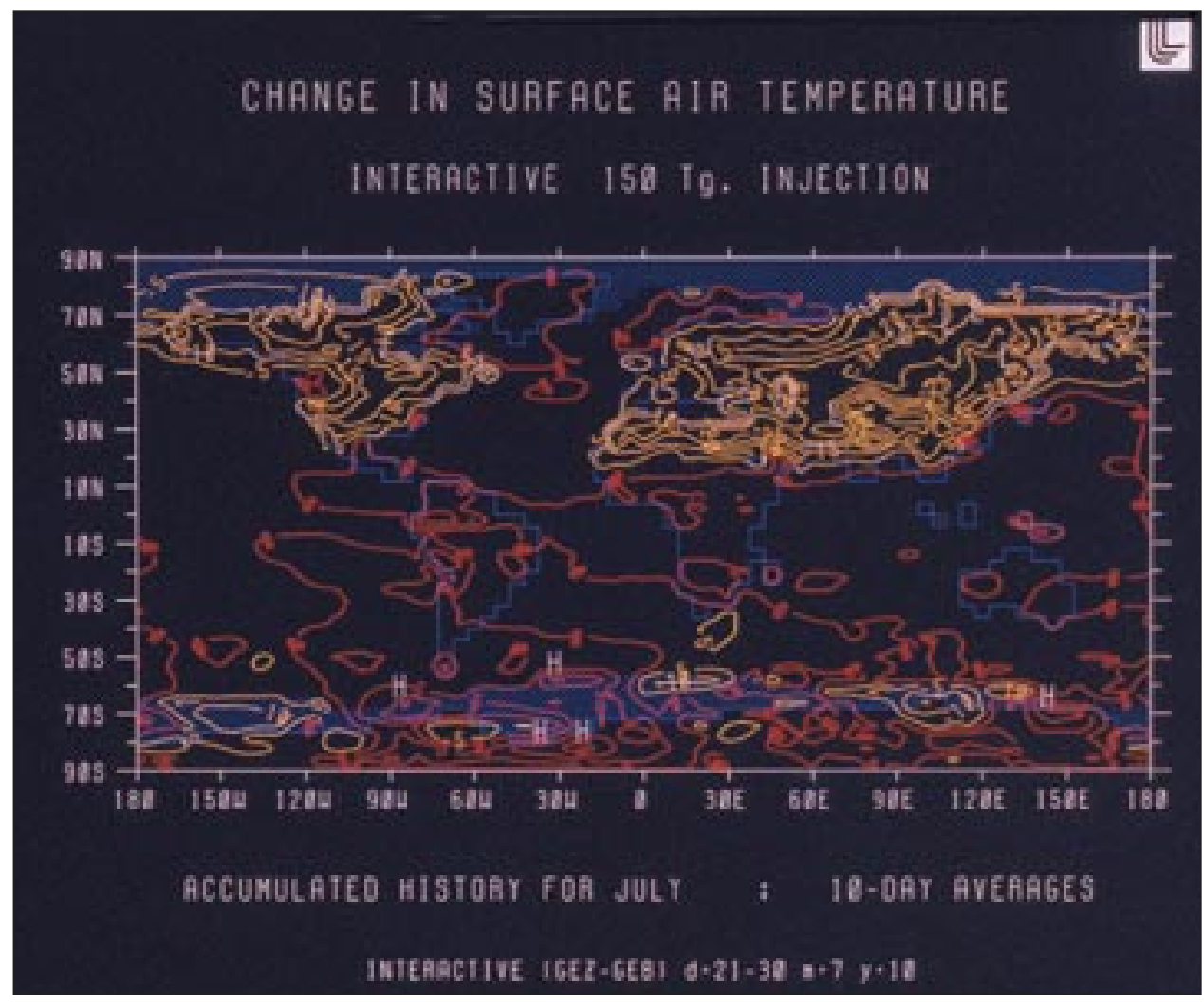




\section{Understanding Past and Future Climate Changes: LLNL's Recent Contributions}

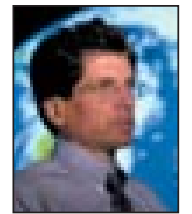

\author{
Phil Duffy \\ Group Leader for Climate and Carbon \\ Cycle Modeling, \\ Energy and Environment Directorate
}

In the 1990s, important progress was made in understanding the causes of 20th-century climate changes and in improving our ability to predict future climate changes. One of LLNL's early contributions during this period was the recognition that aerosol particles from human activities are an important agent of climate change. Including the effects of aerosols in climate models allowed these models, for the first time, to simulate the temperature history of the 20th century with reasonable accuracy. This greatly increased the credibility of future-climate projections made with these models. In the mid-1990s, scientists at LLNL and elsewhere showed for the first time that at least some of the warming that occurred during the 20th century was caused by humans. This "detection of anthropogenic climate change" moved global warming from a theoretical prediction to an observed fact. Subsequent work has shown that the signature of human activities can be seen in meteorological variables other than surface temperature.
Through the Program for Climate model Diagnosis and Intercomparison, LLNL has been a leader in performing systematic evaluation of climate model results. This has led to better understanding of the shortcomings of climate models and ultimately to better climate models. More recently, LLNL scientists have developed one of the first comprehensive coupled climate/carboncycle models. Climate change affects the natural processes that add and remove carbon-dioxide from the atmosphere. Since carbon-dioxide is an important greenhouse gas, this means that there are potentially important feedbacks between the climate system and the carbon cycle. Coupled climate/carbon-cycle modeling will allow us to directly simulate the climatic impact of proposed changes to energy policy that affect carbon emissions.

Finally, using Advanced Simulation and Computing (ASCI-)scale computer resources, LLNL scientists have recently performed global climate simulations at a much finer level of spatial detail than ever before. This high-resolution modeling has allowed improved simulation of climate on a regional spatial scale and improved assessment of the societal impacts of climate change.

\section{CCM3@T42}

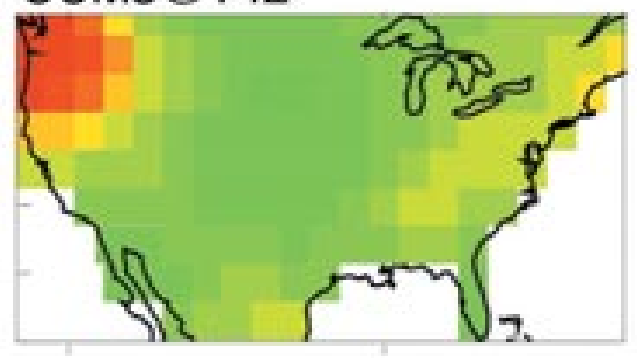

CCM3@T170
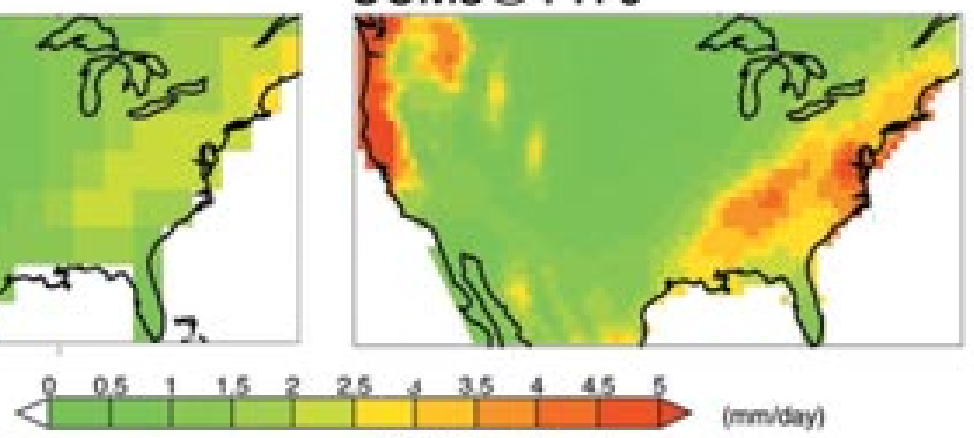

CCM3@T239

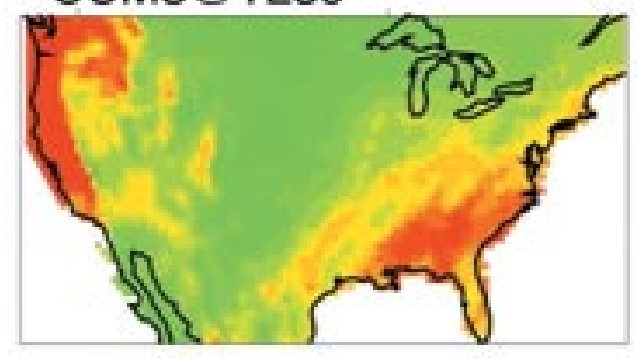

\section{VEMAP observations}

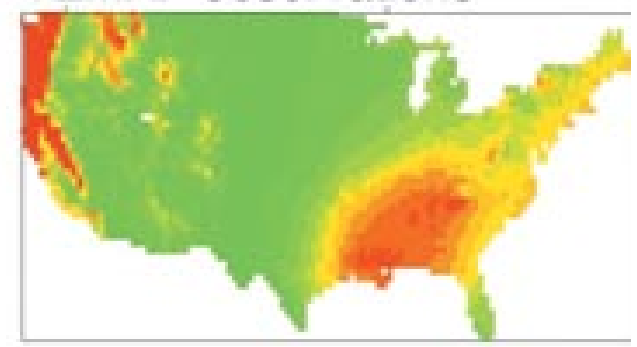

Using terascale computing resources at Livermore and DOE, LLNL scientists have shown that increasing the "resolution" (degree of detail) in climate models produces more realistic simulations of the present climate. The figure shows wintertime precipitation in the USA as simulated by a climate model at resolutions of $300 \mathrm{~km}$ (upper left), $75 \mathrm{~km}$ (upper right), and $50 \mathrm{~km}$ (lower left). Also shown are observed precipitation rates (lower right). As the model resolution is increased, the simulated precipitation agrees more closely with observations. 


\section{Astrophysics At Livermore}

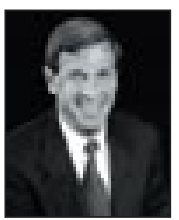

\section{Bruce Tarter}

Associate Director at Large and

former Director
Because of the strong scientific relationship between the phenomena that occur in a nuclear weapon and in astronomical environments, astrophysical research has always been a natural extension for many of our programmatic tools and methods. From the earliest days, the Laboratory has often been a leader in introducing new computational techniques, in studying the underlying physics at high temperatures and densities, and in pioneering experimental work under those extreme conditions.

One of the first contributions was the use of the Laboratory's state-of-the-art computers to revolutionize the way stellar-evolution calculations were done. In the mid-1960s two seminal papers appeared, both dealing with the end of a star's life. The first, by Colgate and White, gave a comprehensive description of a supernova explosion and formed the basis for all subsequent work in the field. Colgate will talk about his early work and its extension into the present day. The second, by May and White, was the first calculation of the collapse of a star to form a black hole, and was the foundation for much of the future work on numerical general relativity.

Later in the 1960s, the Lab was very active in the early days of $x$-ray astronomy, with two groups - one led by Seward and one by Mark—each fielding experiments on sounding rockets. As computing power increased, it also enabled more complex simulations, and Laboratory researchers pursued many different problems. Star formation was a major topic, as was the modeling of gases excited by the $x$ and gamma rays now being discovered observationally. And, supernova calculations were pursued aggressively, with Wilson and a variety of collaborators leading the field. Weaver and Woosley also developed a comprehensive code that modeled all the late stages of stellar evolution, with a focus on predicting the formation of nearly the full range of elements in the periodic table.

In the late 1980s and 1990s, a substantial amount of experimental and theoretical work was done on physical processes. Experiments with the Electron Beam Ion Trap provided first-ever atomic data needed for stars and weapons, equation-of-state measurements on Nova and gas guns gave new insights into the behavior of light elements at very high pressure, and the world's benchmark opacity code was developed by Iglesias and Rogers. The work from roughly the late 1960s until the early 1990s will be summarized in my talk, including the role of the Institute for Geophysics and Planetary Physics in catalyzing much of the research in astrophysics.

Recent work includes two major observational efforts and the extension of the supernova research into three-dimensional computations. Max's talk will discuss her decade-long work in applying adaptive optics techniques to optical telescopes and the great enhancements in "seeing" that this has made possible. The MACHO project, lead by Alcock, identified the amount of dark "ordinary" matter in our galaxy, and represented the first detection of gravitational lensing. Finally, Dearborn will summarize the state of the art in supernova research. 


\section{Explosions at Livermore, or Explosions I Have Known and Don't Understand, Including Supernovae}

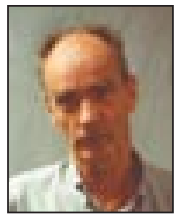

\author{
Stirling Colgate \\ Senior Fellow, \\ Los Alamos National Laboratory
}

LLNL started with designing nuclear weapons and testing nuclear weapons-even LANL's, i.e., the 15-megaton Bravo test. Holms and Narver had to learn that the Earth is round, but we had to learn why a kiloton fireball was focused down the diagnostic pipelines to the block house, two miles from point zero. From the prior calculations of the diagnostic backgrounds for the Morgenstern test, we should have predicted what later went wrong. Always there were explosions more complicated than we could understand. Montgomery Johnson and I were supposed to understand nuclear explosions in space. Would a thin layer of the case blow off at $\mathrm{MeV}$ energies, even relativistic velocity? Wouldn't a supernova do the same thing and make the cosmic rays of space? And so we published-not knowing. The Russian delegation at Geneva for the test negotiations were supercapable scientists. They scoffed at the suggestion that a supernova might trigger a simple treaty-monitoring detector and start a nuclear war. (We needed legitimate spy satellites and they didn't.) The Russian refrain, "Who knows what supernova will do?" started a second career for me. Edward Teller funded Dick White and me to "damn well know what a supernova will do" so that we would not be so dumb next time-good luck. But we did have good luck with the suggestion that the neutron star would emit its energy in neutrinos in a black-body spectrum, an outlandish suggestion at the time and so we published. The supernova 1987A saved the day (and night, too). A spherically symmetric neutrino-diffusive supernova will not work. It must be nonaxisymmetric. Why? "Based on the turn of a plausible phrase, thickening the erudite verbal haze, based on the summary, hiding the mummery, and demolished the theory that Jack built."

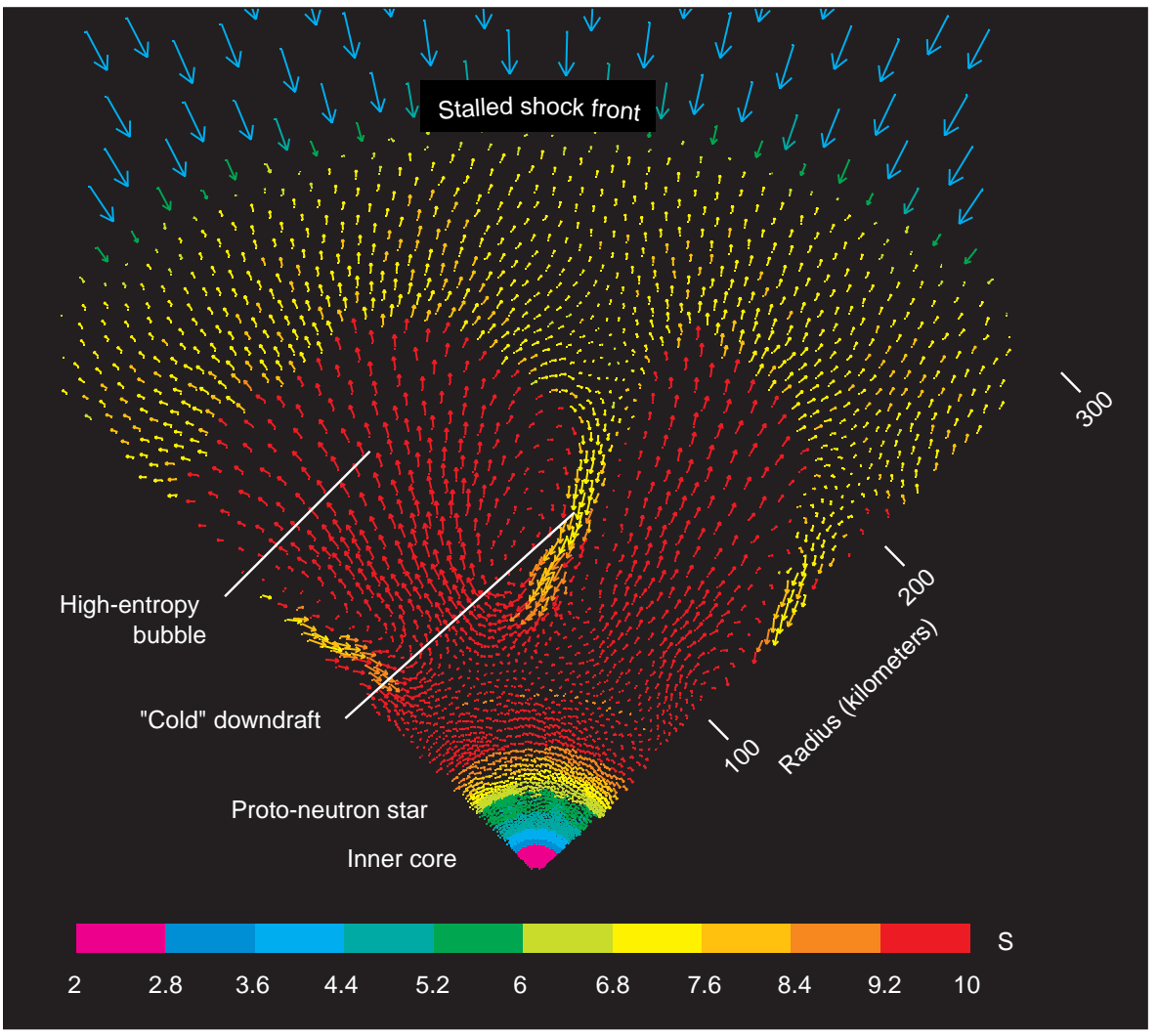

The first calculations (in two dimensions), by Mark Herant, of the first neutrino-driven supernova explosions that really worked. Here, large-scale convection is driven by the heat transported by neutrinos. The colors are arcane properties-like entropy-of the explosion-driven matter, derived from temperature and density. The preservation of the large scale of the plumes, essential for an explosion, is an inherent property of a relativistic gas, i.e., heated by neutrinos adjacent to the neutron star. 


\section{Highlights of the Seventies and Eighties}

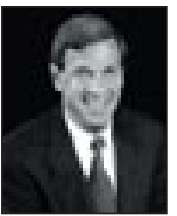

\section{Bruce Tarter}

Associate Director at Large and former Director
Astrophysical highlights of both theoretical and experimental work at Livermore from the early 1970s through the 1980s will be discussed.

Experimental topics include early work with sounding rockets for $\mathrm{x}$-ray observations (as part of that era's readiness program), laboratory astrophysics, instrumentation for space missions (including the Clementine mission), and the devel- opment of the MACHO program. Theoretical subjects include the role of the Laboratory in large-scale numerical simulation and in physical data areas such as equation of state and opacities. The formation of the Institute for Geophysics and Planetary Physics, and the organization of the Laboratory's astrophysical work and its relationship to the general community will also be discussed.
In 1994, the space probe Clementine carried cameras and other equipment developed by the Laboratory. Imaging in 13 different spectral bands, Clementine's cameras mapped $99.9 \%$ of the lunar surface.

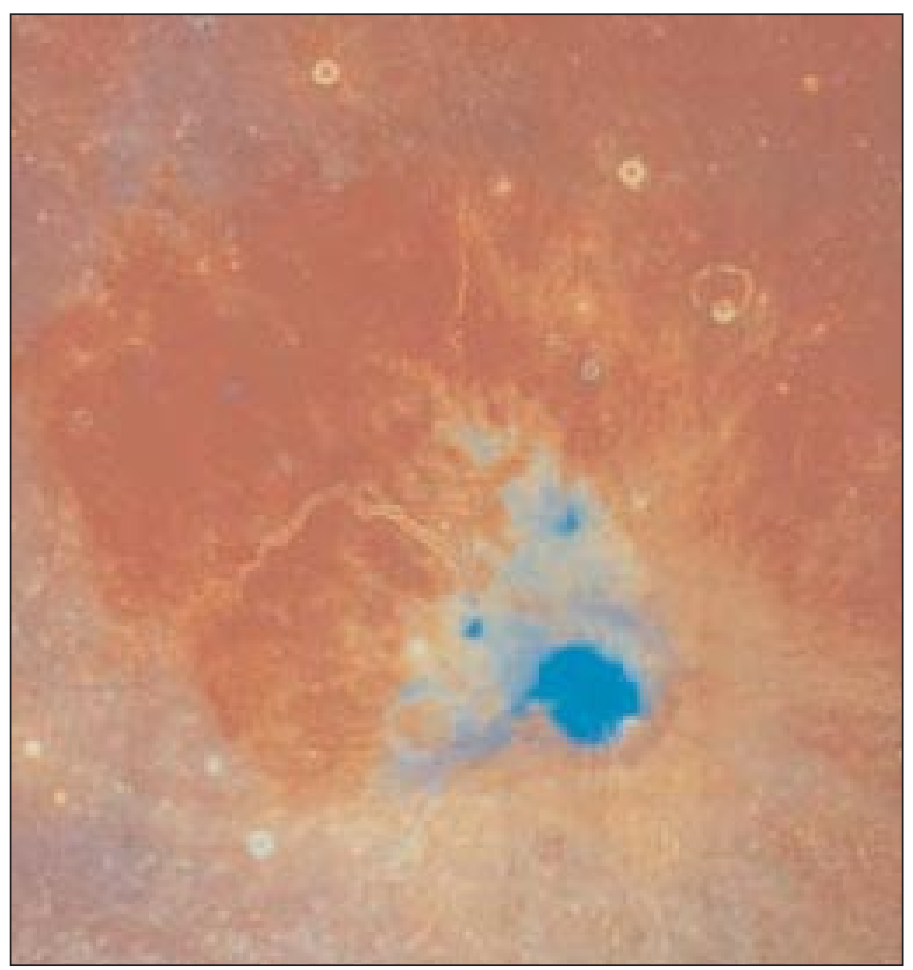




\section{Adaptive Optics: A Sharper View of the Universe}

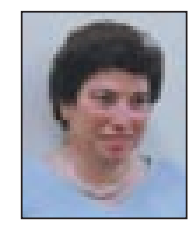

\section{Claire Max}

Physicist,

Physics and Advanced Technologies Directorate

and Director, Center for Adaptive Optics,

University of California, Santa Cruz

Adaptive optics (AO) is a technology for removing image blur, so that a telescope or microscope can "see" at its theoretical limits. First suggested by an astronomer in the 1950s, the technology was initially developed for military applications and for use within high-powered laser beams. Adaptive optics has undergone a genuine renaissance in the last decade for astronomical applications. LLNL has played a key role at the Lick and Keck Observatories, developing their laser guide stars and contributing to their AO systems. This talk will begin with an overview of $\mathrm{AO}$ methods and will then discuss recent exciting astronomical discoveries made using the Keck and Lick Observatory AO systems.

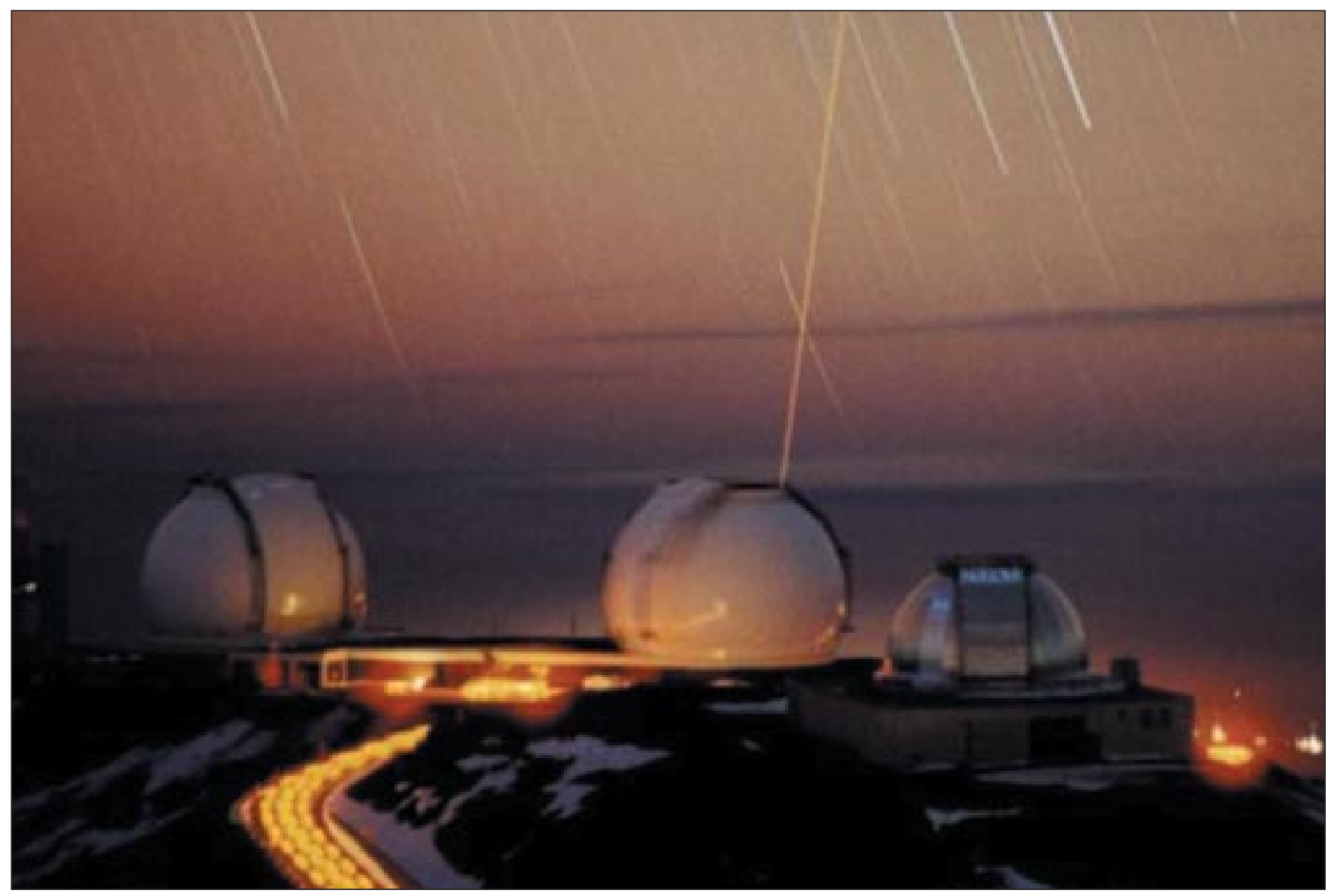

A very faint beam from the Keck Observatory sodium laser appears in this 20-minute exposure. The laser creates a "virtual" star high above the Earth's surface, which is not visible to the human eye but is bright enough to guide high-resolution AO at Keck. In this image, taken from 600 meters away, hazard lights from a vehicle mark the steep descent path of the summit, and the motion of the Earth has created star trails in the sky. 


\section{The True Heart of a Star}

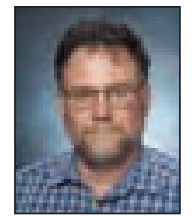

\section{David Dearborn}

Research Physicist,

V Division,

Physics and Advanced Technologies Directorate
Stars are much more than faint objects twinkling in the night sky. They provide the fundamental quantitative measures for the universe, setting the scale(s) for distances, and limits on the age. Stars are also used as physics laboratories, constraining atomic, nuclear, and particle properties and providing observational signatures that challenge our understanding of complex physical processes. Current practices in stellar evolution employ one-dimensional calculations that apply only to a minority of the observed stars (single nonrotating stars, or well-detached binaries). Even in these systems, astrophysicists are dependent on models of complex three dimensional (3-D) processes like convection. With the advent of massively parallel computers and the development of codes to use them, LLNL was uniquely situated to begin understanding stars without this artificial limit. Early results of modeling whole stars in 3-D will be presented.
A slice through the heart of a four-solar-mass star shows the extended region where nuclear burning drives convection. This mixing has long-term affects on the evolution of this star, with observable consequences.

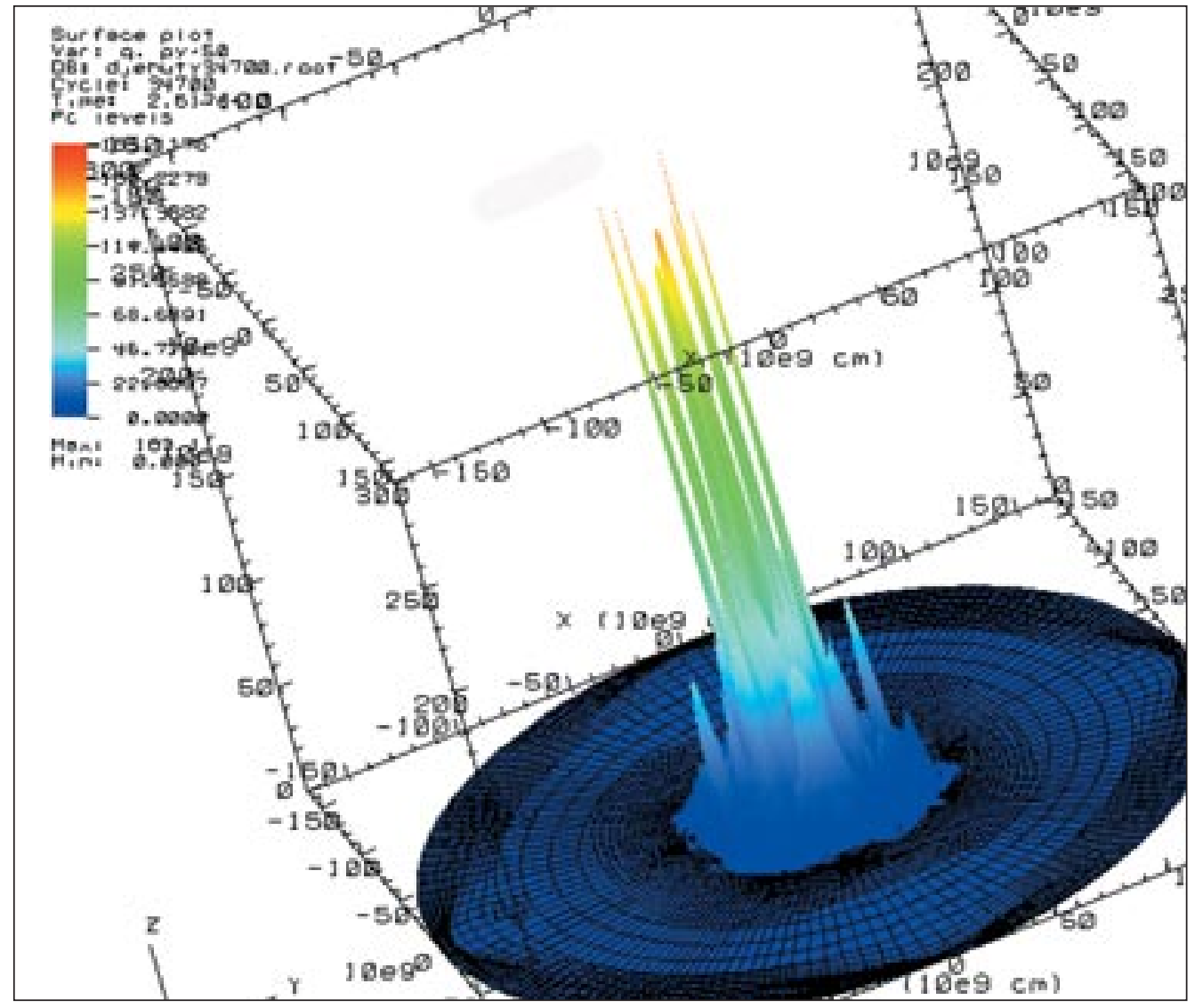




\section{Session Overview Inertial Fusion and High-Energy-Density Research at LLNL}

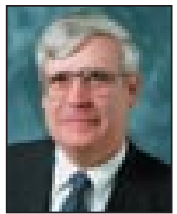

George Miller

Associate Director,

National Ignition Facility Programs

LLNL has been at the forefront of fusion research over the past five decades. Beginning with early programs to produce energy densities similar to those found in nuclear-weapons explosions, LLNL's fusion-energy programs began exploring paths to fusion-energy production using both magnetic confinement, e.g., mirror machines and tokamaks, and inertial confinement using highenergy lasers.

Literally within days of the invention of the laser in 1960, scientists at LLNL, including John Nuckolls, Stirling Colgate, and Ray Kidder, proposed using lasers to inertially confine and implode capsules containing deuterium and tritium (DT) fusion fuel to produce ignition and burn. Nuckolls and Colgate conceived the so-called indirect-drive approach to fusion using capsules in hohlraumsthin-walled metal cylinders that behave in many respects like x-ray "ovens" to ablate the capsule surface and implode the DT fuel. Kidder explored direct-drive approaches, in which symmetrically arranged lasers directly illuminate the fusion capsule and implode it.

Beginning in the 1970s, LLNL produced a series of larger and more energetic solid-state lasers using flashlamp-pumped neodymium-doped glass. Research on these lasers led to breakthroughs in power conditioning and delivery systems, optics, crystals, laser glass, and optomechanical systems designed to produce extremely high-quality laser beams suitable for imploding fusion targets. In addition, sophisticated control systems and diagnostics provided high-quality physical data in support of theoretical modeling of laser performance, laser-materials interactions, plasma physics, and high-energy-density physics. These data have provided key insights into the conditions that exist inside planetary cores, stars, and exploding nuclear weapons.

Today LLNL is leading the way to inertial confinement fusion with the National Ignition Facility (NIF). NIF will be the worlds largest and most energetic laser system and is designed to produce the conditions now understood to be necessary to create fusion ignition and burn with energy gain. NIF, with its 192 laser beams housed in a stadium-sized facility, is nearing completion; the first four laser beams of NIF_- "first light" - will be commissioned in the coming year. Soon afterwards, the first experiments will begin concurrently with the completion of NIF's remaining laser beams over the following four years.

Today's session, Fusion Research at LLNL, will provide a look at the past, present, and future of laser research, inertial fusion and high-energydensity science, and the prospects for inertial fusion energy for our nation's future energy security. 


\section{Early Steps toward Inertial Confinement Fusion}

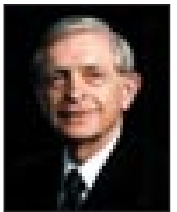

John Nuckolls

Director Emeritus

In the decade after Los Alamos detonated the first thermonuclear explosion, inertial confinement fusion (ICF) concepts were calculated at Livermore, lasers were demonstrated at Hughes and elsewhere, and a small Livermore laser-fusion program was started to explore weapons-physics applications. There were three key questions: (1) What is the smallest possible fusion explosion? (2) How can a small fusion explosion be initiated in the laboratory? and (3) What are the practical applications of small fusion explosions?

Early calculations showed that deuterium-tritium masses smaller than 1 milligram could be sufficiently symmetrically and stably imploded to thousands of times liquid density. These implosions would generate tens of pounds (TNT equivalent) of fusion yield, if we could invent a way to generate hundreds of times higher than chemical energy densities to drive miniradiation implosions, i.e., a non-nuclear primary. We considered pulsed power, hypervelocity guns, plasma guns, chargedparticle accelerators. Within a year, lasers were invented. Directly driven implosion/burn calculations were made.

Net energy could be generated provided we could invent ways to make the microfusion orders of magnitude more efficient than the state. Concepts were identified to meet this challenge. However, it was estimated we would need a 106-joule laser. The first decade of ICF ended with great long-range challenges. After a decade of worldwide progress in the development of lasers and declassification of simple microfusion calculations, the outlook for ICF changed dramatically. In the early 1970s, the Atomic Energy Commission approved the Laboratory's modern ICF Program.

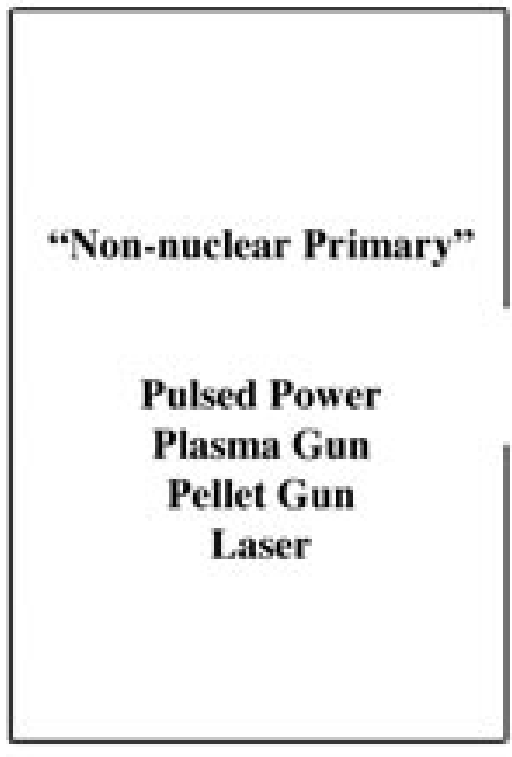

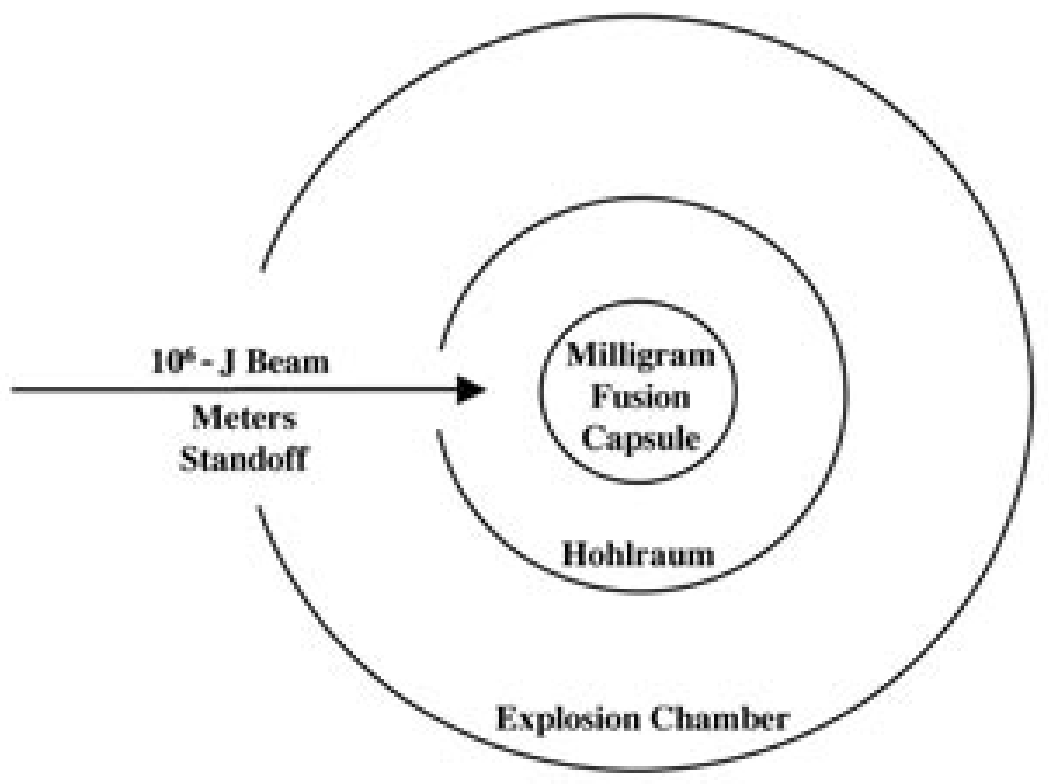

(not to scale)

In this early inertial confinement fusion scheme, a milligram of deuterium is imploded to superhigh densities in a low-temperature minihohlraum energized by a beam from a non-nuclear primary. 


\section{High-Energy-Density Physics, with Applications to Astrophysics}

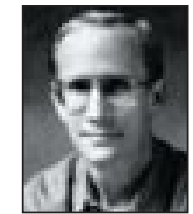

Bruce Remington

Group Leader, High-Energy-Density

Physics Program,

National Ignition Facility Programs

Directorate
High-energy-density (HED) physics broadly refers to the study of collections of matter under extreme conditions of pressure, temperature, and density. The experimental facilities used for these studies are high-power lasers, magnetic-pinch generators, and charged-particle beams. The HED physics pursued on these facilities is still in its infancy, yet a broad user community has nucleated, and new regimes of experimental science are emerging. Examples drawn from astrophysics include work relevant to supernovae and supernova remnants, astrophysical jets, radiatively driven molecular clouds, accreting black holes, planetary interiors, and gamma-ray bursts. The talk will review a selection of science highlights in this field from the past decade and provide a look ahead to the coming decade.

\section{Supernova 1987A simulation}

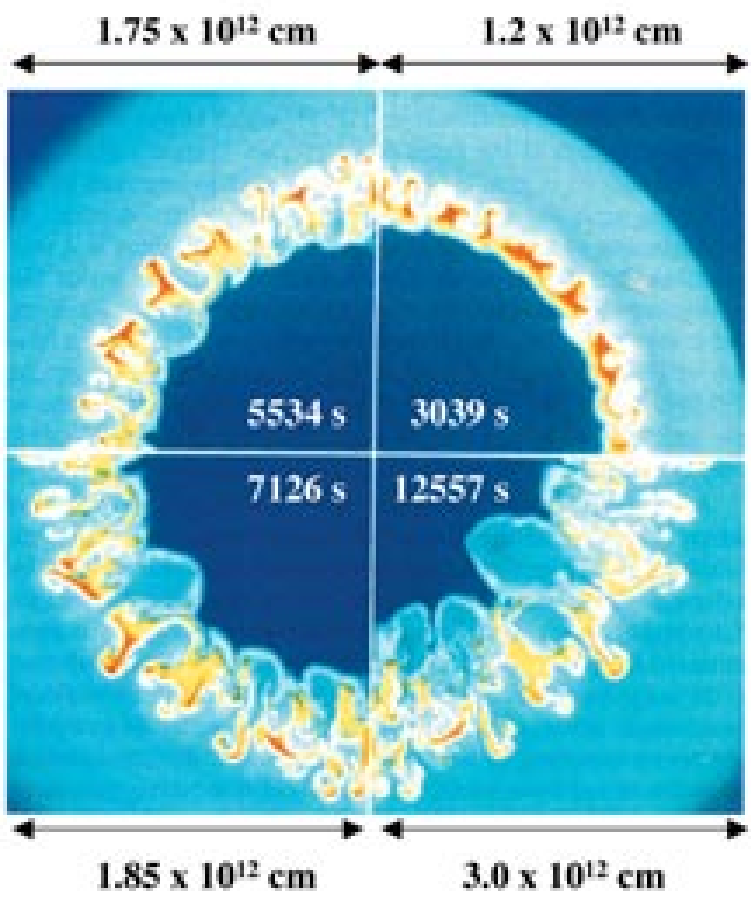

\section{Laser experiment}

(Nova laser shot 29012016)

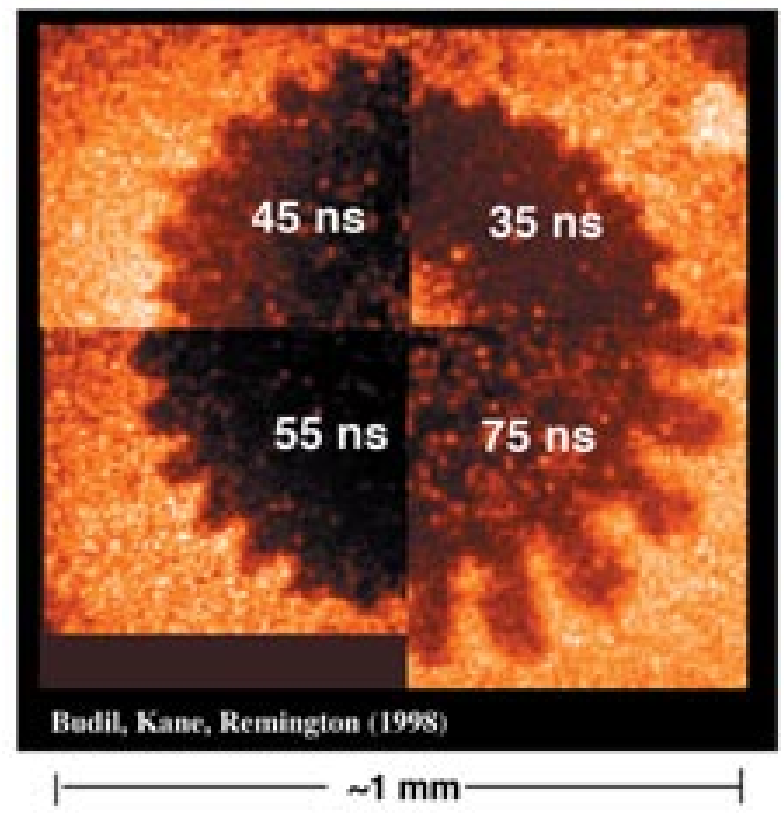

Scaled hydrodynamics relevant to supernova explosions can be reproduced in strong shock-driven laser experiments. Here, the spatial scales differ by 13 orders of magnitude and the time scales by 11 orders of magnitude, but the turbulent hydrodynamics are the same. 


\section{Development of the Scientific Foundation of Inertial Confinement Fusion}

\author{
John Lindl \\ Fusion Energy Program Leader, \\ Physics and Advanced Technologies Directorate \\ and Chief Scientist for the Ignition Program, \\ National Ignition Facility Programs Directorate
}

Achieving controlled fusion in the laboratory has been one of the grand challenges of the past 50 years. With the invention of the laser in 1960, researchers around the world realized that a powerful new tool would become available to pursue this goal, using inertial confinement of a hot, compressed plasma. Over the past 30 years, LLNL has developed a series of lasers, along with extensive diagnostic and theoretical capabilities, that have allowed us to play a leading role in this approach to fusion. Achieving ignition and burn with inertial confinement using a megajoule-scale laser driver requires achieving deuterium-tritium fuel densities of 1,000 to 10,000 times liquid density. Getting to this goal entails development of 100 -megabar pressures, the achievement of $1 \%$ uniformity in the pressure, and the implosion of shells, which are inherently unstable, to velocities of 300 to 400 kilometers per second. This talk discusses some of the critical research that led to an understanding of how to achieve these requirements and presents some of the possibilities for the application of this work to fusion energy production.

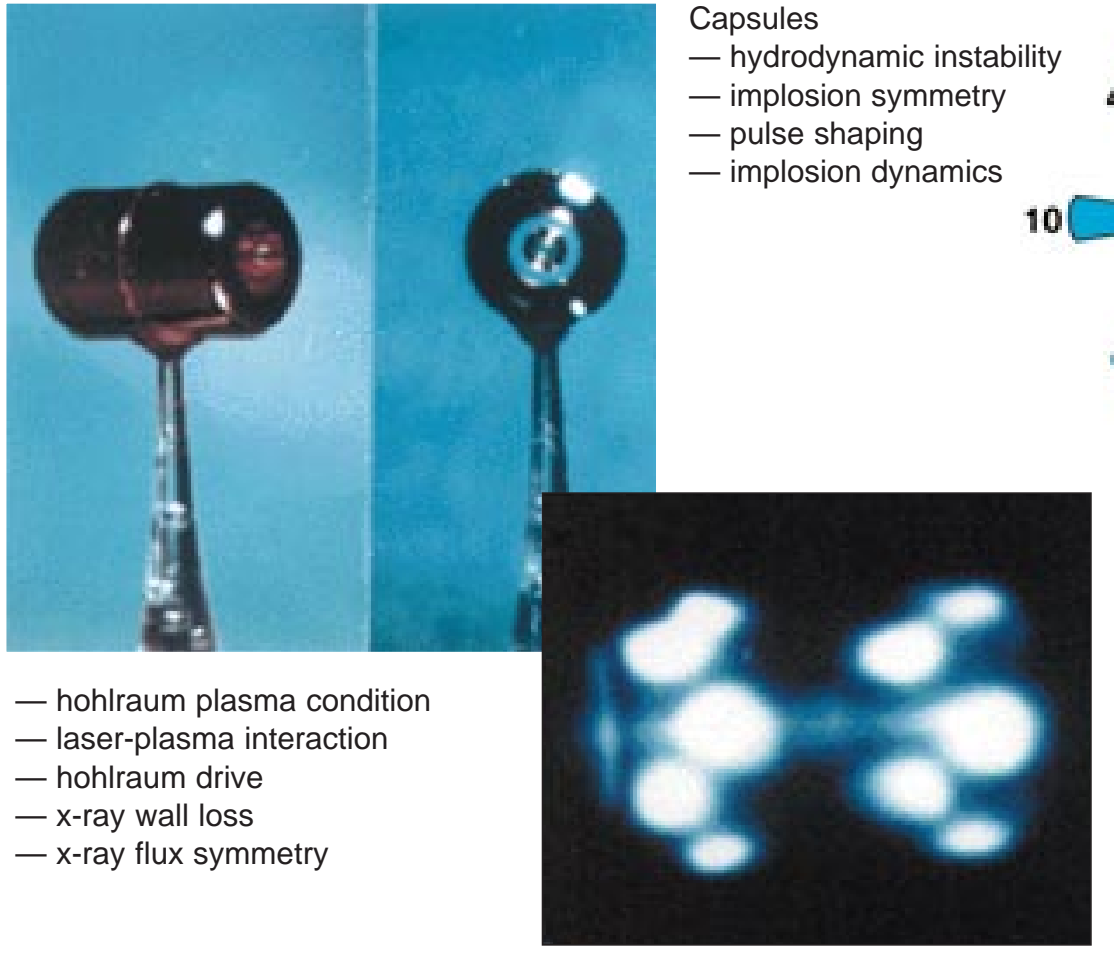

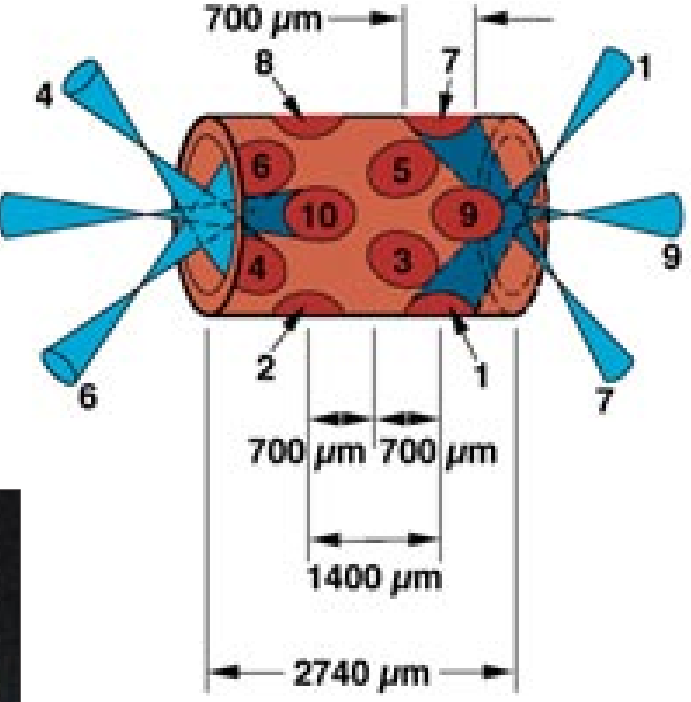

3- $\mu$ m-thick tungsten hohlraun 10-beam irradiation Laser energy $\sim 18 \mathrm{~kJ}$ (total) Wavelength $=0.35 \mu \mathrm{m}$ Pulse length $=1 \mathrm{~ns}(\mathrm{FWHM})$

The Nova ignition physics program utilized hohlraums and capsules, which were scaled to test key issues. 


\section{Inertial Fusion and High-Energy-Density Science on the National Ignition Facility}

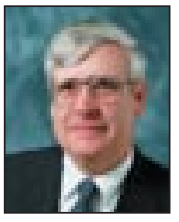

\section{George Miller}

Associate Director,

National Ignition Facility Programs

Inertial fusion and high-energy-density science worldwide is poised to take a great leap forward. In the U.S., the National Ignition Facility (NIF), currently under construction at LLNL, is nearing its "first light" milestone. NIF is a football stadiumsized laser and experimental facility containing a 1.8megajoule, 500-terawatt, 351-nanometer laser system. When completed, NIF will be the world's largest laser system, providing a center to study inertial confinement fusion and the physics of extreme energy densities and pressures.

In NIF, up to 192 energetic laser beams will compress small fusion targets to conditions where they will ignite and burn, liberating more energy than is required to initiate the fusion reactions. NIF experiments will allow the study of physical processes at temperatures approaching 100 million Kelvin and 100 billion times atmospheric pressure. These conditions exist naturally only in the interior of stars and in nuclear-weapons explosions.

In the course of designing the world's most energetic laser system, a number of significant technology breakthroughs have been achieved. Research is also underway to develop short-pulse capability on NIF. High-energy petawatt beams can provide more efficient backlighter diagnostic applications and fast ignitor capability. This presentation will discuss the technology challenges and solutions that have made NIF possible, along with enhancements to NIF's design that could lead to petawatt and even exawatt power levels. The talk will also discuss the upcoming experimental program on NIF, including next year's experimental commissioning activities.

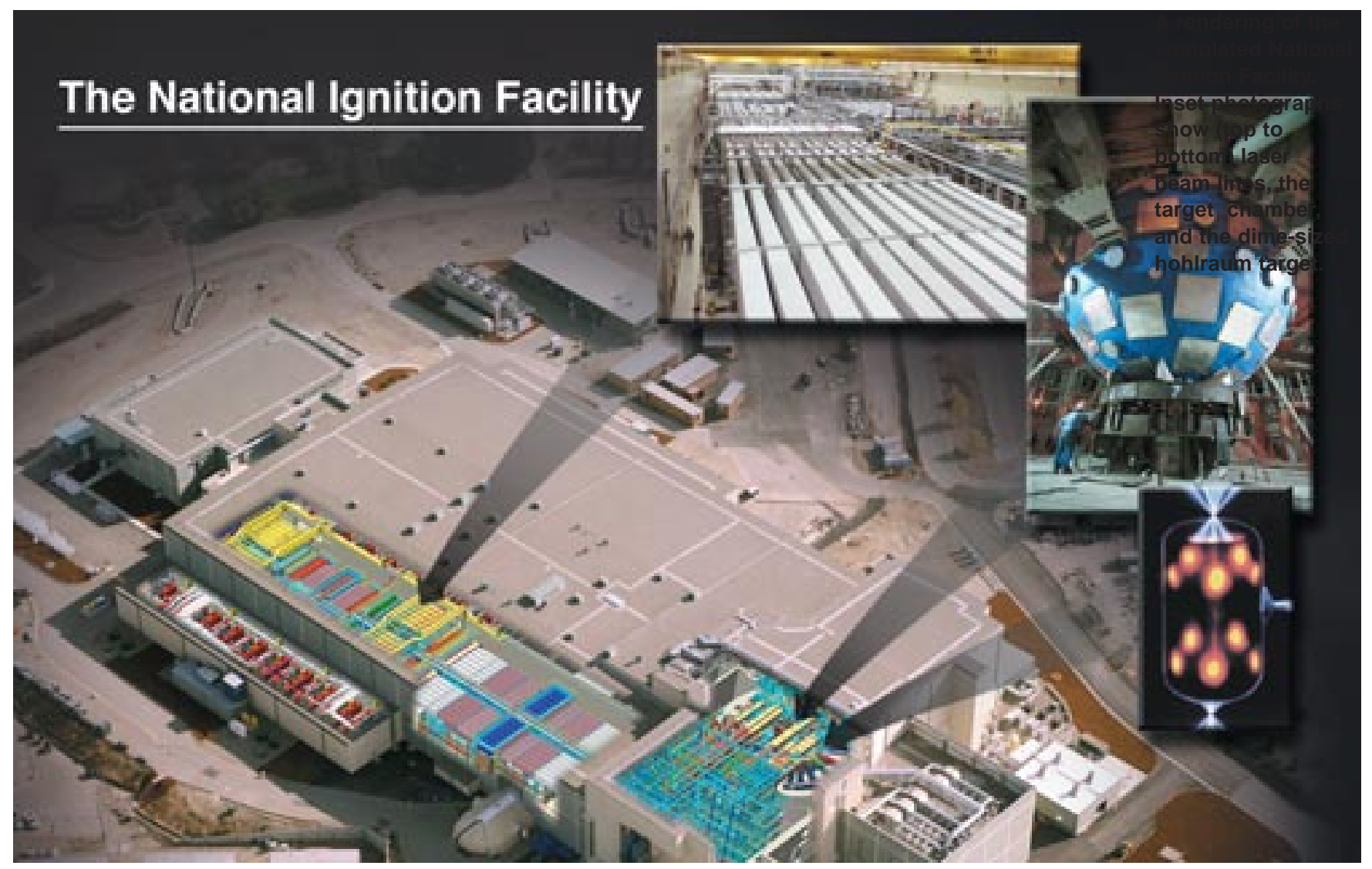




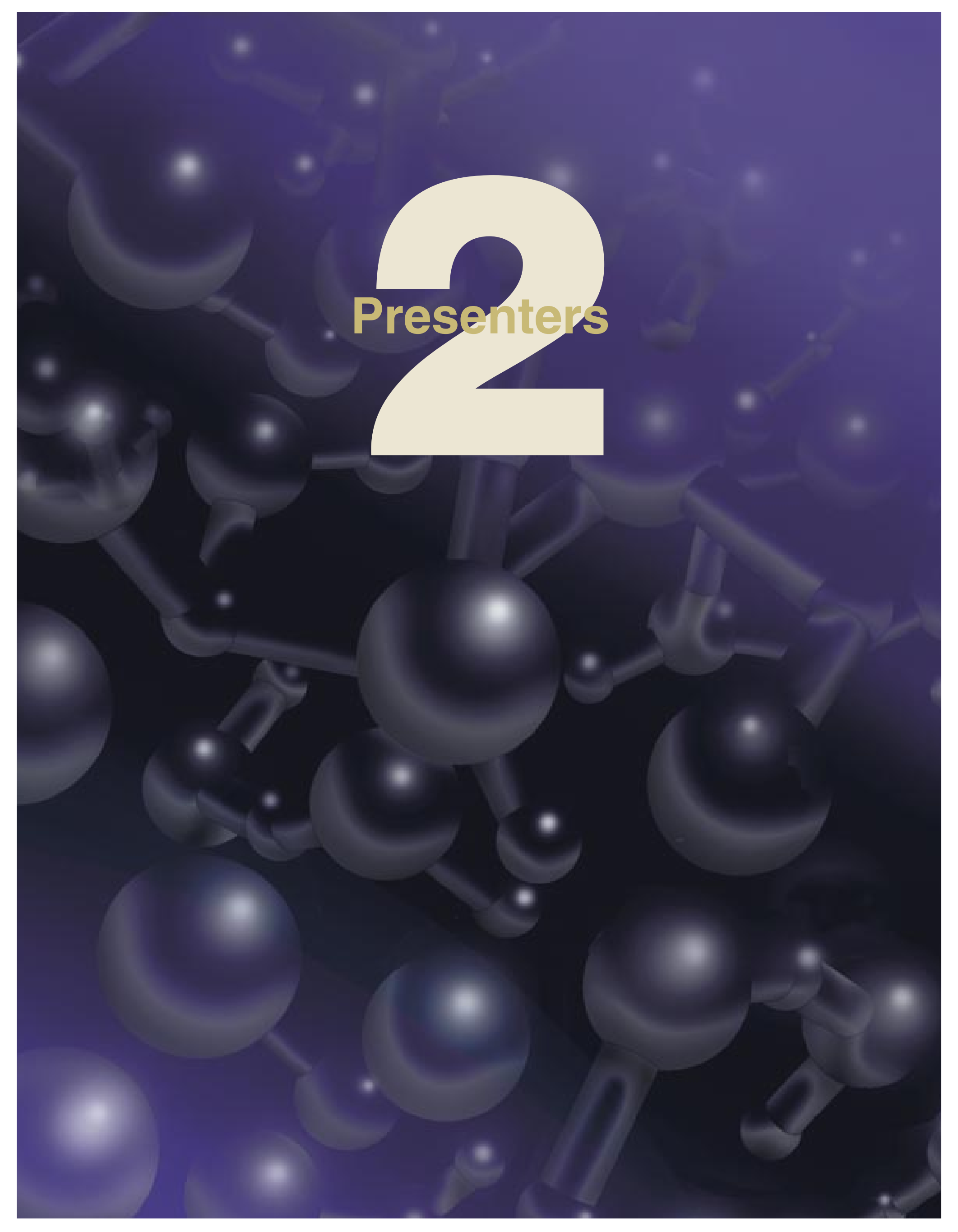




\section{Stirling A. Colgate}

Senior Fellow,

Los Alamos National Laboratory

Stirling Colgate has been a staff physicist at LLNL and is now at Los Alamos National Laboratory (LANL). From 1952 to 1954 he led diagnostic testing of nuclear weapons in the Pacific. In 1954 he initiated the helical magnetic fusion program at Livermore; and in 1959 he served as scientific advisor to the U.S. State Department in Geneva for negotiations with the USSR for the cessation of nuclear testing. From 1965 through 1974, he was president of New Mexico Institute of Mining and Technology. He was elected to the National Academy of Science in 1985 and in 1990 he received the Rossi prize for work in astrophysics, having predicted in 1963 the emission of neutrinos from supernovae, recently observed from the nearby Supernovae 1987A. He is a Senior Fellow at LANL and a Fellow of the American Physical Society. He has published over 250 papers in magnetic fusion, astrophysics, atmospheric physics, and inertial fusion. Stirling Colgate also works in the fields of volcanic explosions, reactor safety, underground nuclear waste storage, underground stress engineering, tornadoes, vortices, myopia, AIDS epidemiology, and human origins. He holds over 20 patents, including diesel engines and cryogenic machinery.

\section{David S. P. Dearborn}

Research Physicist,

V Division

Physics and Advanced Technologies Directorate

David Dearborn, currently a research physicist at LLNL, has held positions at the Copernicus Institute in Warsaw, the Institute of Astronomy in Cambridge, the California Institute of Technology, and Steward Observatory in Tucson, AZ. He has worked extensively in astrophysics (nucleosynthesis, stellar evolution, and astro-particle physics),

archaeoastronomy (research on the Inca as well as editing a newsletter and journal), nuclear-weapons design and testing, and re-entry vehicle flight testing. He is now the principal investigator on a project to develop a three-dimensional stellar evolution and structure code for astrophysical research in a massively parallel environment. He has received two Dudley awards for research in the History of Astronomy, three Weapons Recognition of Excellence awards from the Department of Energy and was the 1998 Shelby Fellow of the Australian Academy of Science. He holds a Ph.D. from the University of Texas at Austin.
Philip B. Duffy

Group Leader for Climate and Carbon Cycle

Modeling,

Energy and Environment Directorate

Philip Duffy is LLNL's Group Leader for Climate and Carbon Cycle Modeling in the Energy and Environment Directorate. He has A.B. and Ph.D. degrees in Astrophysics from Harvard and Stanford, respectively. Since 1986 he has been at LLNL, where he has worked on strategic defensive systems studies, modeling of atomic physics, atmospheric spectroscopy, and climate modeling. In climate modeling, he has worked on evaluating and improving models of the ocean circulation. Most recently, he has investigated the effects of increasing resolution in models of the ocean and atmosphere. His current interests include application of climate models to assessment of the societal impacts of climate change.

\section{Joe W. Gray}

Professor of Laboratory Medicine and Radiation Oncology,

University of California, San Francisco

Joe Gray, former bioscientist and section leader at LLNL from 1972 to 1991, is Professor of Laboratory Medicine and Radiation Oncology at the University of California, San Francisco (UCSF), where he also is Program Leader for Breast Oncology and Cancer Genetics in the UCSF Comprehensive Cancer Center. In addition, he is Principal Investigator of the Bay Area Breast Cancer Translational Research Program, a Specialized Program of Research Excellence project sponsored by the National Cancer Institute. His main research interests at UCSF involve elucidation of the genomic and genetic events that are associated with breast and ovarian cancer development and progression. His goals are to use the resulting information to better predict individual response to therapy and to develop novel gene-targeted therapeutics. He holds a Ph.D. in Physics from Kansas State University and is the recipient of many awards, including the E.O. Lawrence Award (1986) and the Exceptional National Service Award (1997), both from the Department of Energy. Dr. Gray is a Fellow of the American Association for the Advancement of Science. 
Cecil E. Leith, Jr.

Senior Scientist,

A Division,

Defense and Nuclear Technologies Directorate

At LLNL from the beginning in 1952, Chuck Leith began working on numerical models of the atmosphere in 1960, in order to understand better the flow patterns that determine the climate. He was the first to create hemispherical atmospheric climate models using the Livermore Advanced Research Computer. In addition to his climate modeling research, he also taught courses in the Physics Department of the University of California (UC), Berkeley and the graduate Department of Applied Science, Livermore of UC Davis. Chuck Leith moved to the National Center for Atmospheric Research (NCAR) in Boulder CO in 1968, where he continued work on turbulence and convection in the atmosphere and their proper treatment in numerical models. In 1969, he became head of the NCAR Dynamics Department.

Returning to LLNL in 1983, he carried out research on numerical simulation of turbulent mixing and applications of massively parallel computers. After retiring in 1991, he has continued to work in climate modeling and simulation as a Participating Guest. Chuck Leith holds A.B. (1943) and Ph.D. (1957) degrees in mathematics from the UC Berkeley.

\section{John D. Lindl}

Fusion Energy Program Leader, Physics and Advanced Technologies Directorate and Chief Scientist for the Ignition Program, National Ignition Facility Programs Directorate

John currently leads the Fusion Energy Program at LLNL and is the Chief Scientist for the Ignition Program in the NIF Programs Directorate. A Fellow of the American Physical Society and of the American Association for the Advancement of Science, he was awarded the Edward Teller Medal in 1993, and in 1994 received the E.O. Lawrence Award for work in inertial fusion. He is the recipient of the 2000 Fusion Power Associates Leadership Award. John received a Ph.D. in Plasma Physics from Princeton University in 1972 and has spent his career at LLNL developing the science of inertial confinement fusion (ICF). Among his positions at LLNL, John led an extensive multiyear effort on the Nova laser, which developed the physics basis for inertial fusion ignition. Prior to that, he was the XDivision leader responsible for theory and modeling in the ICF Program as well as Advanced
Applications of ICF, including inertial fusion energy. John's book, Inertial Confinement Fusion (SpringerVerlag, 1998), has become a widely used reference for the science of inertial fusion.

\section{Michael C. MacCracken}

Senior Global Change Scientist,

Office of the U. S. Global Change Research Program (on assignment from LLNL)

Michael MacCracken is on assignment as senior global change scientist from LLNL to the interagency Office of the U.S. Global Change Research Program (USGCRP) in Washington, DC. From 1993 to 1997, Mike served as executive director of USGCRP, which is charged with helping to coordinate the combined research efforts of ten federal agencies to understand and improve predictions of climate variability and change, depletion of stratospheric ozone, and the long-term, global-scale impacts of humans on the environment and society. From 1997 to 2001, Mike served as executive director of the National Assessment Coordination Office, a USGCRP-sponsored activity to assess the potential consequences of climate variability and change for the U.S., and was coordinating author for the chapter on impacts and adaptation for the US Climate Action Report 2002. Mike has been closely involved in the official U.S. Government review of the assessment reports prepared by the Intergovernmental Panel on Climate Change (IPCC), and has been a co-authorcontributing author of various chapters in their reports. Mike received his B.S. in engineering from Princeton University in 1964 and his Ph.D. degree in Applied Science from the University of California, Davis/Livermore in 1968. Mike then joined LLNL's Physics Department. Mike was the deputy division leader of the Atmospheric and Geophysical Sciences Division from 1974 to 1987 and division leader from 1987 to 1993, before accepting the Washington assignment. He is a fellow of the American Association for the Advancement of Science.

\section{Claire E. Max}

Physicist,

Physics and Advanced Technologies Directorate and Director, Center for Adaptive Optics, University of California, Santa Cruz

Claire Max graduated from Radcliffe College (A.B.) and Princeton University (Ph.D.) in Astrophysical Sciences. After working as a postdoc in the Physics Department at the University of California (UC), Berkeley, she came to LLNL, where has done research 
in laser-plasma interactions, computer simulation of astrophysical plasmas, and adaptive optics. Max was the founding director of the Institute of Geophysics and Planetary Physics at LLNL, and later served as the Director of University Relations at the Laboratory. She is a member of the American Academy of Arts and Sciences, and a Fellow of the American Physical Society and the American Association for the Advancement of Science. Max's current research interests include adaptive optics and laser guide stars, and their use for studies of the Solar System and active galactic nuclei. In addition to her work at LLNL, Max holds a joint appointment in the Astronomy Department at UC Santa Cruz.

\section{Mortimer L. Mendelsohn}

Senior Scientist and former Associate Director, Biology and Biotechnology Research Program

Mort Mendelsohn, senior scientist and former associate director of the Biology and Biotechnology Research Program at LLNL, received his M.D. degree from Harvard University Medical School (1948) and his Ph.D. from Cambridge University (1958). From 1958 to 1972 , he was a professor at the University of Pennsylvania. He came to LLNL in 1972, where he worked as the associate director of the Biomedical \& Environmental Research Program until 1992. After retiring from LLNL, he became the director at large and vice chairman of the Radiation Effects Research Foundation in Hiroshima, Japan. Since 1995, Mort Mendelsohn has served as senior scientist at LLNL.

\section{Fred P. Milanovich}

Director Emeritus, Chemical / Biological National Security Program, R Division,

Nonproliferation, Arms Control, and International Security Directorate

Fred Milanovich received his B.S. in Physics from the United States Air Force Academy in 1967 and a Ph.D. in Applied Physics from the University of California, Davis in 1974. He is a founder and former Program Leader for the Chemical/Biological National Security Program (CBNP) within the Nonproliferation, Arms Control, and International Security Directorate at LLNL. The CBNP is providing an integrated response to the emerging threat of chemical and biological terrorism with innovations in detection technology, bioinformatics, fate and transport analyses, and incident response. Milanovich has published extensively and holds many patents for optical sensors and measurement instruments. His research interests include trace biodetection, laser spectroscopy, analytical instrumentation development, and microtechnology.

\section{George H. Miller}

Associate Director,

National Ignition Facility Programs

As associate director for NIF Programs at LLNL, George Miller provides integrated leadership to the senior management team directing the NIF Program and has been a major participant in the development of the Department of Energy's Stockpile Stewardship Program. He received his B.S. (1967), M.S. (1969), and Ph.D. (1972) degrees in Physics, all from the College of William and Mary. From 1972 to 1980, he served as a physicist in A Division, and became A-Division leader and program leader for all thermonuclear design and computational physics development. In 1984, he was named deputy associate director for Nuclear Design, and in 1985 he was named associate director. He spent 1989 in Washington as the special scientific advisor on weapons activities to Secretary of Energy Admiral James D. Watkins. George Miller holds memberships in the American Physical Society and Sigma Pi Sigma. He has received awards and honors from the National Science Foundation Graduate Fellowship, Gulf-General Atomics Fellowship, and Sigma Pi Sigma. He is a member of the USSTRATCOM Strategic Advisory Group and the Navy Steering Task Group.

\section{John H. Nuckolls \\ Director Emeritus}

John Nuckolls is Director Emeritus of LLNL. A physicist, he developed advanced weapons concepts for the U. S. nuclear deterrent and ballistic missile defense systems during the Cold War. He is a pioneer and leader in the development of laser fusion energy. He served as associate director for Physics from 1983 to 1988, and as director of the Laboratory from 1988 to 1994 during the transition to the post-Cold War era. As director, he strongly advocated programs to address the emerging threats of rogue states and terrorists armed with weapons of mass destruction. John is a member of the National Academy of Engineering, and a Fellow of the American Physical Society and the American Association for Advancement of Science. He is a consultant to the Department of Defense and a member of the USSTRATCOM Strategic Advisory 
Group. He served as advisor to the Director of Central Intelligence (1989-1999). He has received awards and citations from the President and Atomic Energy Commission; the Secretary of Energy; the Secretary of Defense; the American Physical Society; the international fusion energy community; and the University of California.

\section{Bruce A. Remington}

Group Leader, High-Energy-Density Physics Program, National Ignition Facility Programs Directorate

Bruce Remington received a B.S. in Mathematics from Northern Michigan University in 1975 and a Ph.D. in Physics from Michigan State University in 1986. He joined LLNL as a postdoctoral associate in 1986 doing nuclear physics research and became a permanent staff physicist in the Laser Programs Directorate in 1988. As a group leader in the High-Energy-Density Physics Program in the NIF Directorate, he initiates and manages experiments on the Omega laser related to high-energy-density regimes, materials science, and astrophysics.

\section{Lisa J. Stubbs}

Acting Director, Genome Regulation and Function Division, Biology and Biotechnology Research Program

Lisa Stubbs is the acting director of the Genome Regulation and Function Division in the Biology and Biotechnology Research Program and a senior staff scientist at the DOE Joint Genome Institute in Walnut Creek, CA. Lisa received her Ph.D. in molecular biology from the University of California, San Diego and received postdoctoral training in molecular genetics and genomics at the California Insitute of Technology, Pasadena, CA and at the European Molecular Biology Laboratory in Heidelberg, Germany and the Imperial Cancer Research Fund in London, England. From Europe, Lisa moved to Oak Ridge National Laboratory in 1990 to head a research group in mouse genetics and genomics before moving to LLNL, where she has been for the past five years.

\section{Bruce Tarter}

Associate Director at Large and former Director

C. Bruce Tarter is the associate director at large at LLNL and was the eighth director to lead the Laboratory since it was founded in 1952. A theoretical physicist by training and experience, he began his career at the Laboratory in 1967. As director from December 1994 through June 2002, he led the Laboratory in its mission to ensure national security and apply science and technology to the important problems of our time. Tarter received a bachelor's degree in Physics from the Massachusetts Institute of Technology and a Ph.D. from Cornell University. He is a Fellow of the American Physical Society, received the Roosevelts Gold Medal Award for Science (1998), the Secretary of Energy's Gold Award (2002), and the NNSA Administrator's Award for Distinguished Service (2002). 


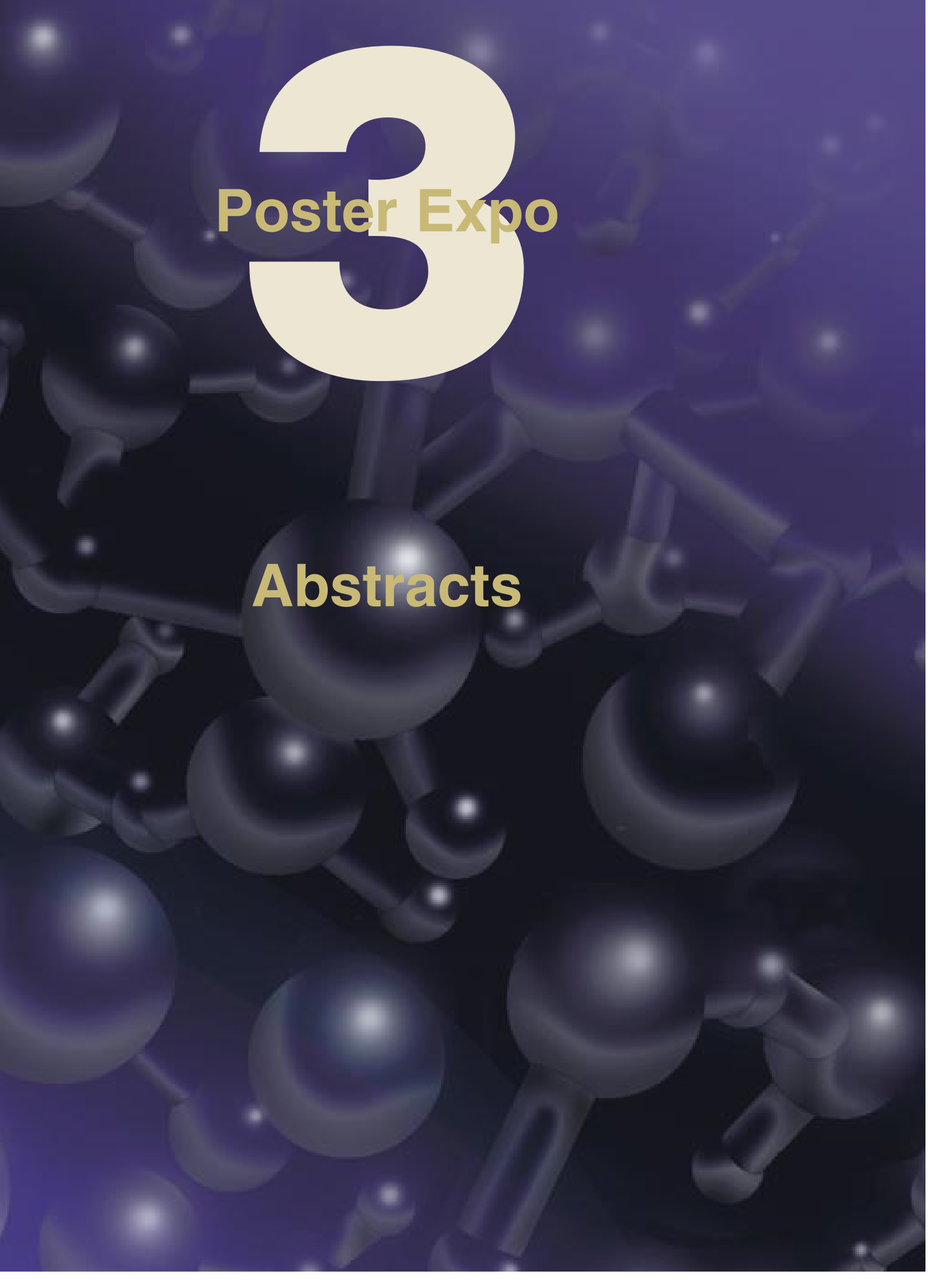


Active Interrogation of Cargo Containers

Arden Dougan et al.

This project, supported by Laboratory Directed Research and Development funding, uses a fully integrated calculational and experimental approach to determine the ultimate performance potential of active-neutron interrogation for detecting weapons of mass destruction (WMD), including chemical weapons materials, high explosives, and special nuclear materials (SNM) in cargo containers. Chemical weapons materials and high explosives can be detected using neutron activation. However, a filled container presents problems: nuisance alarms may arise from conflicting signatures, identifying some elements may require long counting times, and the density of the container contents will attenuate or moderate both the source and emitted neutrons. For shielded SNM, active interrogation relies on the detection of signatures from induced fission of the material.

Current active-interrogation measurement techniques-prompt fission, delayed fission, differential die-away, and delayed-fission gamma emission-pose challenges. This project explores the boundaries of feasibility of active-neutron interrogation by mapping out the limits of the method using carefully chosen experiments together with extensive, high-fidelity modeling. This combined approach of experiments and calculations will enable us reach to a broad and solidly grounded understanding of performance that is not achievable with experiments or calculations alone.

\section{Adaptive Optics in Astronomy and on Earth}

Bruce Macintosh et al.

Adaptive optics (AO) - the rapid control of the phase of light-is a key technology for a wide range of applications. Much original development was driven by astronomy-the need to take sharp images of distant objects in spite of the distortions introduced by the Earth's turbulent atmosphere. LLNL has built an AO system for the 3-m Shane telescope at Lick Observatory, equipped with the world's only operational laser guide star to generate an artificial reference beacon anywhere in the sky. We also helped build the AO system for the 10-m W.M. Keck telescope, the world's largest, which now routinely achieves resolutions better even than the Hubble Space Telescope. Next- generation $\mathrm{AO}$ systems are under development for even larger telescopes or for difficult tasks such as imaging planets orbiting other stars. Adaptive optics has applications to a wide variety of projects. In collaboration with the National Science Foundation Center for Adaptive Optics, we are developing $\mathrm{AO}$ systems to study human vision. Adaptive optics can cancel out the distortions introduced by the optics in the eye to produce super-sharp vision or to allow microscopes to image the invididual cells in a living human retina. LLNL's I Division is also applying $\mathrm{AO}$ to high-data-rate laser communications and remote surveillance. Ultimately, compact, next-generation $\mathrm{AO}$ systems will be a feature of almost any advanced optical system.

\section{Advances in High-Performance Computing at LLNL}

Bing Young

For the past 50 years, computing and super computing at LLNL have enabled scientific advancement in support of the national-security mission. In order for researchers to better understand predict complex physical phenomena, our computation teams have pushed the limits of the largest, fastest, most powerful computers in the world.

The Laboratory's first supercomputer was the Remington-Rand Univac-1. Between April 1950 and February 1957, it is estimated that the Univac executed as many calculations as 440 human "calculators" could perform in 100 years, if they worked 40 hours per week, 52 weeks per year with no mistakes. Today's ASCI White machine, produced by IBM, is rated at a peak performance of 12.3 TFLOPs. We've improved computer processing power by 10 orders of magnitude in 50 years, and it is reasonable to suggest that computing can improve another 10 orders of magnitude in the next 50 years. This poster illustrates the Laboratory's rich computing history, our present strengths, and the possibilities for the future. 
Applications of Ion-Beam Analytical Techniques to Environmental and Biomedical Research at the Center for Accelerator Mass Spectrometry

John Knezovich et al.

The Center for Accelerator Mass Spectrometry (CAMS) at LLNL is the most versatile and productive facility of its kind in the world. The center houses AMS systems that provide exceptionally sensitive approaches for measuring concentrations of specific isotopes [e.g., carbon-14 $(14 \mathrm{C})]$ in relatively small $(<1 \mathrm{mg})$ samples and a nuclear microprobe that provides micrometerscale resolution of elements in biological materials. The sensitivity and precision of AMS has lead to a variety of applications in environmental and biological research, much of which is conducted in collaboration with university researchers. For example, 14C-AMS has been successfully used to determine the history of earthquakes, the dynamics of the global carbon cycle, and the distribution and transformation of chemicals in humans. Other isotopes have similar uses in earth and biosciences as well as nationals security applications.

\section{Applied Structural Genomics of Human and Microbial Proteins}

Matthew A. Coleman, Peter T. Beernink, and Brent W. Segelke

With the quantity of sequence data provided by the Human Genome Project and other sequencing efforts, the challenge becomes how to understand and make use of this information. The Human Genome Project has revealed how little we really know of biology, as most of the genes discovered have no known function or homology to known genes. Characterizing the proteins encoded by these genes through informatics or large-scale experimental initiatives, called proteomics, is the new frontier in biological research. A number of pilot proteomics projects are underway using high-throughput structural genomics, but they have all run into significant bottlenecks-protein expression, purification, and crystallizationbecause the majority of proteins do not express well and intrinsically have only moderate solubility, making them inadequate candidates for structural studies. Our approach is to develop rapid, cell-free protein- expression testing and functional analysis, which enables us to select targets from a collection of many proteins. We also use protein engineering to modify the inherent solubility of identified targets and pull many more proteins through to our highly efficient crystallization screening protocol (CrysTool). Further optimization of the process is ongoing, and we have developed an efficient, automated crystallization facility, with an established throughput of $>1000$ crystallization experiments per day.

\section{Autonomous Pathogen Detection System (APDS) Offers Rapid and Continuous Monitoring of Multiple Airborne Biological Threat Agents}

Steve Brown et al.

The Autonomous Pathogen Detection System (APDS) is a stand-alone pathogen-detection system capable of rapid, continuous, low-cost environmental monitoring of multiple airborne biological threat agents. The primary focus of the APDS has been on protection of civilians from terrorist attacks; however, the same system could also have a role in protecting military personnel from biological warfare attacks. APDS instruments can be used at high-profile events such as the Olympics for short-term, intensive monitoring or more permanent installation in major public buildings or transportation nodes. The APDS has several key advantages over competing technologies: (1) the ability to measure up to 100 different agents and controls in a single sample; (2) the flexibility and ease with which new beadbased assays can be developed and integrated into the system; (3) the presence of an orthogonal, realtime detection module for highly sensitive and selective nucleic acid amplification and detection; (4) the ability to use the same basic system components for multiple deployment architectures; and (5) the relatively low cost per assay ( $<\$ 1$ per 10 -plex or $\$ 0.10$ per assay) and minimal consumables.

\section{Biological and Materials Science Applications of Single-Molecule Spectroscopy}

Chris Hollars et al.

Single-molecule optical spectroscopy is a powerful tool for investigating a variety of systems. Research using single-molecule optical spectroscopy over the past decade has revealed unique photophysical phenomena that have enhanced our understanding of molecular 
interactions. Our research group uses singlemolecule detection and spectroscopy to study and characterize systems that have applications in biological and materials sciences. In the biological sciences, we use surface-enhanced Raman spectroscopy (SERS) to better understand the biophysical properties and interactions of biomolecules such as DNA and proteins. We are also developing instrumentation and experimental schemes aimed at ultrasensitive pathogen detection using single-molecule fluorescence colocalization. Once completed, this system will allow detection of femtomolar quantities of a specific target molecule.

Our research into materials focuses on polymer materials and nanocrystals (quantum dots). The unique properties of certain polymers allow them to emit light and may have applications in ultrathin LED displays. The fluorescent and electroluminescent properties of these polymers are dictated by the electron delocalization within the polymer. Our results indicate a solvent dependent effect on the shape and, therefore, the electron delocalization found in these polymers. Finally, in collaboration with other research groups, we are using the single molecule techniques to better understand the luminescent properties of selected nanocrystals and their potential use as markers in biological applications.

\section{Biophotonics for Medicine}

Duncan Maitland et al.

Biophotonics is the technology of generating and using light and other forms of radiant energy whose quantum unit is the photon for visualization, measurement, analysis, and manipulation of biological materials. Biophotonics exploits the high spatial resolution, sensitivity, and spectral specificity of photonics to understand biologic structure and function, a major topic of research and development at universities and throughout industry. Applications and research can range from fluorescence identification of single biomolecules and viruses, to the use of light to image and selectively treat tumors. We strongly believe that photonics-based systems will be widely available, cost-effective, and ultimately used to screen for predisposition to diseases and, in some areas, provide noninvasive therapies.
Together with other discipline groups and directorates at LLNL, M Division has led a partnership with University of California, Davis to obtain a National Science Foundation Center for Biophotonics Science and Technology (CBST). The poster highlights some of the M-Division biophotonics research that will be included as part of the CBST. Also, biophotonics applications in the treatment and diagnosis of stroke are featured as an example of the potential impact of biophotonics on a specific disease.

\section{BlueGene/L-The Next Generation of Scalable Computing Platform}

Mark Seager, Lynn Kissel, and Kim Yates

BlueGene/L is a next-generation massivelyparallel computing system designed for research and development into computational science targeted at selected applications of interest to the Advanced Simulations and Computing (ASCI) Tri-Lab community and its University Alliance partners. A select but broad set of science application areas have been identified as an initial focus for execution on BlueGene/L. BlueGene/L is a scalable ultra-computer targeted for $216=$ 65,536 compute nodes, not counting hot spares and software development platforms. Each node consists of a single-compute ASIC and SDRAMDDR memory chips. The nodes are interconnected through multiple networks, one of which connects the nearest neighbors mapping the nodes into a $64 \times 32 \times 32$ three-dimensional torus. The ASIC that powers the nodes is based on IBM's system-on-a-chip technology giving a very compact, low-power building block that is being used to create an extremely high-computedensity system with very attractive cost performance. BlueGene/ $\mathrm{L}$ is a cellular architecture in that the 512-node basic building block of the system can replicated in a regular pattern, with no introduction of bottlenecks as the system is scaled up.

\section{Computational Characterization of Biomolecules}

Daniel Barsky et al.

This poster surveys our recent applications of molecular dynamics and homology modeling, emphasizing how our calculations are making a difference in biology. Given a protein structure, we are using molecular dynamics simulations to 
study the change in plasticity of that structure upon changing one amino acid residue. For example, we have looked at several variants (mutants) of the DNA repair enzyme Ape1 that are associated with human disease. Through simulation, we are predicting exactly how these structurally less stable proteins will likely have a reduced repair capacity, and thus lead to disease. We also use and develop homology-modeling methods to predict the structure and function of a given protein from its gene sequence by comparing it to proteins of known structure with a high level of sequence similarity. To help understand the virulence of pathogens such as Yersinia Pestis, the bacterium that causes plague, we have used homology modeling to predict the function and structure of a very low-homology $(\sim 13 \%)$ protein that is a suspected virulence factor of $Y$. Pestis, and have used the same methodology to create models of complexes of the rad51 DNA repair proteins.

\section{Continuum Solvent Model for First-Principles Molecular dynamics simulations} Jean Luc Fattebert and Francois Gygi

Many important chemical and biochemical reactions occur in aqueous solutions. Firstprinciples molecular dynamics simulations of such processes are very expensive since a large number of solvent molecules must be explicitly introduced in the calculation.

As an alternative, simplified models that represent only the average effect of the solvent on the solute can be used. We have developed a new continuum model that represents the electrostatic effects of a polar solvent as a dielectric medium surrounding the solute. Compared to other existing models, it is better suited for plane waves electronic structure calculations and molecular dynamcis. This model has been integrated into the code JEEP developed at LLNL allowing first-principles molecular dynamics simulations of organic molecules in aqueous solution.

\section{Critical Skills Internships-Science \& Technology Education Program}

Don Correll and Barry Goldman

Internships for college students as Laboratory employees working in the National Nuclear Security Administrations (NNSA) mission-based science research are an important element of the
Laboratory's attraction and recruitment workforce strategies. With NNSA direct funds, the Science and Technology Education Program (STEP) administers internships for college students who are entering careers important to the critical skills workforce needs of the Laboratory's NNSA Programs. Upon graduation, approximately 15\% of the STEP interns from the last two fiscal years (FY01 and FY00) were recruited into full-time positions within LLNL. STEP's eleven internship projects in FY02 have attracted approximately 100 college student employees (undergraduate and graduate) in four disciplines: chemistry and materials science, computer science, engineering, and physics. STEP college interns are given the opportunity to have their LLNL research accomplishments listed on the STEP student portfolio Web site. Information within each student portfolio includes the intern's expected graduation date, grade point average, and e-mail address.

\section{Development of Synthetic High-Affinity Ligands \\ Julie Perkins}

The development of a synthetic high-affinity ligand (SHAL), a small, synthetic molecule with high affinity and specificity for a target protein, has the potential for making a significant impact in the areas of cancer therapy and biowarfare. For example, using a SHAL to target the delivery of a radionuclide specifically to the site of nonHodgkins lymphoma could more effectively treat that metastatic disease than currently available methods. A SHAL targeting the clostridial neurotoxins (tetanus and botulinum), which are widely recognized as potential biowarfare threats, could be integrated into a sensory device. The process of producing a SHAL combines biology, computational techniques and organic and physical chemistry.

\section{Distribution of Spore Particles and Decontamination Agents in an Office Environment \\ Paula Krauter et al.}

Biological warfare agents (BWA) risks are a function of effective dispersion, exposure and infectivity mitigation efforts, and the public's perception of risk. A critical concern of indoorenvironment BWA decontamination is effective 
deployment of the decontamination agent on the surfaces where BWA settles. An exercise was conducted at the U.S. Army Dugway Proving Ground (DPG) to (1) measure the BWA contamination levels at different locations and on various materials within an office setting, and (2) evaluate liquid-gel oxidizer and paraformaldehyde gas as decontamination agents. This study evaluated the distribution of a Bacillus anthracis surrogate, $B$. subtilis var. niger, also known as B. globigii (BG) in two mock offices, each approximately $64 \mathrm{ft} 2$. Each office was contaminated with $4 \mathrm{~g}$ of BG by a single puff of air using a Dixon disseminator, then the BG was further dispersed with an oscillating fan. The spore powder provided by DPG consisted of single spores and spore clumps ranging up to $10 \mathrm{~mm}$. A swab sample was collected before and after decontamination over a 4-in. ${ }^{2}$ area within each square foot of the office (approximately 400 samples per room). The swab was placed in a sterile, buffered solution containing sodium thiosulfate. Serial dilutions were plated onto TSA agar, incubated at $37^{\circ} \mathrm{C}$ and counted. The detection limit of the analyses was about $102 \mathrm{CFU} / 4$ in.2. Three-dimensional models of the deposition of BG spores on the walls, ceiling and floor of each contaminated office indicated that more spores were on the floor than on any other surface, and the distribution was fairly uniform upon surfaces of the same geometry. Concentrations of surviving $B G$ recovered from the office after decontamination with liquid/gel had about the same mean value as that of the office decontaminated with paraformaldehyde. Both treatments eliminated BG on the floor by about 5 orders of magnitude.

\section{DNA Compaction Induced by the Yeast Mitochondrial Protein ABF2p}

Larry Brewer et al.

Single-molecule studies provide kinetic and mechanistic information that is often obscured in bulk measurements. In our experiments, an optical trap is used to manipulate individual DNA molecules in a novel microfluidic flow cell to study important biological processes that take place in cells. We have observed the compaction of individual molecules of linear dsDNA by the mitochondrial $(\mathrm{mt})$ packaging protein $\mathrm{ABF} 2 \mathrm{p}$ using both fluorescence microscopy and atomic force microscopy (AFM). Using an optical trap to manipulate single DNA molecules attached to beads within a novel two-channel flow cell, we were able to observe that the percentage compaction of each DNA molecule varied inversely with the hydrodynamic drag imposed by the fluid flowing by it. The maximum force exerted by the ABF2p protein during compaction of the DNA was measured to have an upper limit of $0.6 \mathrm{pN}$. This, together with the ABF2p footprint of $28 \mathrm{bp}$, gives an energy associated with compaction of $5.5 \mathrm{pN} \mathrm{nm}$, which is just greater than the thermal energy $k T$. The fact that ABF2p only weakly packages DNA has implications for important mitochondrial processes such as transcription and replication. AFM studies were able to observe how ABF2p bends and compacts single DNA molecules to form nucleoid-like structures.

\section{Effects of Trichloroethylene (TCE) on the Mobilization of Metals in Livermore}

Richard A. Brown

The objective of this project was to determine if trichloroethylene (TCE) at any concentrations would leach measurable masses of zinc or manganese from typical soils in the Livermore Valley of California. TCE, at concentrations from zero to $500 \mathrm{ppm}$ (by mass) in distilled water to pure TCE, was leached through soil samples from the Livermore Valley. After leaching with TCE, the soil samples were subjected to a sequential extraction procedure, developed by soil scientists Tessier and others. Liquid samples from each sequential extraction were collected and were analyzed by flame atomic absorption spectroscopy for concentrations of zinc and manganese remaining in the leachate samples. Higher concentrations of TCE leached out significant amounts of manganese and zinc compared to leaching with distilled water only. These results suggest that TCE, and perhaps other solvents, will leach out quantities of metals from similar sediments and eventually into ground water. This same pattern may occur with other metals that are chemically similar to zinc and manganese, such as cadmium and mercury. The interactions between TCE, and perhaps, other solvents and those metals could result in an increased public health risk in groundwater than could be attributed to the presence of the solvent alone. 


\section{Electromagnetic Imaging of $\mathrm{CO}_{2}$ Sequestration at an Enhanced-Oil-Recovery Site \\ Barry Kirkendall and Jeff Roberts}

The Laboratory is currently involved in a longterm study using time-lapse multiple frequency electromagnetic (EM) characterization at a carbon dioxide $\left(\mathrm{CO}_{2}\right)$ sequestration enhanced-oilrecovery (EOR) site in Lost Hills, California that is operated by Chevron Production Division. Our team combines laboratory and field methods to image a pilot $\mathrm{CO}_{2}$ sequestration EOR site using the cross-borehole EM technique, improves the data-processing techniques in $\mathrm{CO}_{2}$ studies by coupling results with petrophysical laboratory measurements, and focuses on new gas interpretation techniques. Field and laboratory results can provide information on subsurface $\mathrm{CO}_{2}$ detection, $\mathrm{CO}_{2}$ migration tracking, and displacement of petroleum and water over time. This project directly addresses national energy issues by (1) developing field and laboratory techniques to improve in-situ analysis of oil and gas enhanced recovery operations and (2) providing a tool for in-situ analysis of $\mathrm{CO}_{2}$ sequestration, an international technical issue of growing importance.

\section{The Evaluation of Flor-Essence ${ }^{\circledR}$ and Essia ${ }^{\circledR}$ Herbal Tonics using In Vitro and In Vivo Models for Breast Cancer Research}

L. Michelle Bennett and Kristen Kulp

Women who are diagnosed with breast cancer often self-administer complementary and alternative medicine (CAM) to augment their conventional treatments, improve health, or prevent recurrence. Approximately 50\% of patients who use CAM do not inform their physicians. When CAM is discussed, counseling is hindered by large gaps in knowledge about safety, efficacy, and potential drug interactions. We have initiated experiments to understand the consequences of exposure to two herbal tonics, Flor-Essence ${ }^{\circledR}$ and Essiac ${ }^{\circledR}$, widely used by breast cancer patients. Using a battery of in vitro tests, we have determined that although these tonics have antimutagenic activity against the common food mutagens IQ and PhIP, they are also able to stimulate the growth of breast cancer cells. Cell proliferation assays have shown that adding these tonics to breast cancer cell medium at concentrations recommended for human use induces proliferation to levels similar to that observed for estrogen alone. When low levels of the tonics are combined with estrogen a synergistic effect results in more cell growth than would be seen by either the tonics or estrogen alone. We have also found that these tonics stimulate estrogen receptor activation to levels that are not significantly different from the effect of estrogen alone.

\section{Experimental and Theoretical Considerations for Modeling Material Performance in a Nuclear Waste Repository \\ Tammy Summers et al.}

Nickel-based Alloy 22 serves as an extremely corrosion-resistant material for the outer barrier of the waste package that has been designed to contain spent nuclear waste in a repository at Yucca Mountain. Corrosion tests are underway at LLNL to provide data for models that will demonstrate that the waste package will contain the waste for thousands of years. Stress corrosion cracking (SCC) is being investigated as a possible degradation mode, but results to date indicate that a high residual tensile stress is required before SCC is initiated. Processes are being developed to mitigate the residual tensile stress around the final closure weld after the waste package container is filled with the waste. In the current design, a titanium drip shield, which is corrosion resistant in the expected environmentalrange, surrounds the top portion of the waste package to prevent water contact with the waste package for as long as the drip shield remains. Thus, the drip shield and waste package provide defense in depth for nuclear-waste containment.

\section{Experiments to Develop an Energetic, High- Intensity Laser-Based Proton Source}

Pravesh Patel

The Laboratory's 100-TW Janus Ultrashort Pulse (JanUS) laser focuses up to $10 \mathrm{~J}$ of light in a 100-fs pulse to an intensity on target that exceeds $1020 \mathrm{~W} / \mathrm{cm}^{2}$. Recent experiments using the JanUSP laser have created highly collimated, high-flux, energetic beams of protons-a rich source of energetic $x$-rays, electrons, and ions. These proton beams are characterized by their high flux, low divergence angle, short time duration, and long mean free path. Our experiments use these properties to explore 
applications that include ultrafast proton heating of materials to warm dense states and picosecond time-resolved proton radiography of opaque, high-Z materials.

\section{A Geospatial Model for Remedial Design Optimization and Performance Evaluation} Victor Madrid, Zafer Demir, and Steven Gregory

Collecting and interpreting large amounts of spatial data are essential for soil and ground water remediation projects but 2-D mapping techniques are often inadequate for characterizing complex subsurface conditions at contaminated sites. To interpret data from these sites, we developed a methodology that allows integration of multiple, 3-D data sets for spatial analysis. This methodology was applied to the Department of Energy Building 834 Operable Unit at LLNL's Site 300 . Site 300 is contaminated with a nonaqueous phase liquid (NAPL) mixture consisting of trichloroethene (TCE) and tetrakis (2-ethylbutoxy) silane (TKEBS). In the 1960s and 1970s, releases of this heat-exchange fluid to the environment resulted in TCE concentrations up to $970 \mathrm{mg} / \mathrm{kg}$ in soil and dissolved-phase concentrations approaching the solubility limit in a shallow, perched water-bearing zone. A geospatial model was developed using site hydrogeological data and monitoring data for volatile organic compounds (VOCs) and biogeochemical parameters. The model was used to characterize the distribution of contamination in different geologic media, and to track changes in subsurface contaminant mass related to treatment facility operation and natural attenuation processes. Geospatial analysis of monitoring data collected over a three-year period helped generate representative monthly VOC plume maps and dissolved-phase mass estimates, which proved to be invaluable in optimizing and evaluating the remedial design and performance.

\section{Industrial Partnering with Lawrence Livermore National Laboratory}

Catherine Elizondo

Now called the Office of Industrial Partnerships and Commericialization (IPAC), LLNL's formal technology transfer program dates back to 1990. Our history shows the value of LLNL's technologies in influencing the direction of entire industry sectors, stimulating the formation of start-up companies, and making the results of our federal programs available for broad industry use. Livermore's creative inventors and authors whose work forms the basis of our partnerships. As the research world becomes increasingly interconnected, and as the amount of R\&D conducted in the private sector grows, LLNL's tradition of industry collaborations will continue to expand. The success of our partners demonstrates the impact of Laboratory-developed technologies as they are commercialized and appear in the marketplace. As we celebrate the year of LLNL's 50th Anniversary, our partnerships continue to gain momentum. This year's results will show our current partnerships against a backdrop of our history.

\section{Information Security in a Hacker's World John Allen et al.}

What is the better environment for scientific collaboration, a computing environment where security is modeled after Fort Knox or a casino? Most currently deployed cyber defenses are static in nature, having more in common with the fixed defensive emplacements of France's Maginot Line or Hitler's Atlantic wall then with security at the Bellagio or Mirage. Modern cyber attacks have much more in common with the hit-and-run agile tactics of modern warfare, which emphasizes integrated intelligence, communication, and mobility. The LLNL Intrusion Detection and Reaction team is exploring a number of concepts to embed security features into the LLNL IT infrastructure, and to process all the information available in a manner that is applicatble to the tactical defense of a large network. We have the vision that like a casino, cybersecurity should be very strong but not an obstacle to legitimate transactions.

\section{Institutional Publications}

Technical Information Department Team

The Laboratory's achievements in science and technology are reported in a variety of awardwinning institutional publications produced by the Technical Information Department under the direction of the Director's Office. Published ten times each year, Science and Technology Review provides for a broad audience feature stories, and short highlights about the Laboratory's science and technology. The Laboratory issues its Annual 
Report, Institutional Plan, and Laboratory Directed Research and Development (LDRD) Annual Report each year. Intended for a wide audience, the Annual Report highlights the Laboratory's major accomplishments for the previous year. The Institutional Plan includes a top-level description of the Laboratory's mission and the direction and future goals for programmatic and operational activities. The LDRD Annual Report provides a brief summary of each LDRD project at Livermore.

In 2002, two special publications were also issued to celebrate the Laboratory's 50th anniversary: 50 Years of Accomplishments and 50 Years of Stories.

\section{Interactive Visualization of Terabyte-Sized Datasets}

Valerio Pascucci

This poster describes a recent Laboratory Directed Research and Development project and early results on the use of progressive algorithms to enable interactive visualization of terabyte-sized scientific data sets. The algorithms employ compression and an hierarchical representation of data to enable interactive exploration of data on displays ranging from a laptop to a PowerWall.

\section{Laboratory Simulation of the X-Ray Emission from Comets}

Peter Beiersdorfer

The surprise discovery of $x$-ray emission from comet Hyakutake in 1996 has opened a new spectral band for studying comets in the inner Solar System. The x-ray emission is thought to be due to charge exchange between heavy solar wind ions and gases in the head of the comet. To understand charge-exchange-induced $x$-ray spectra and to develop their diagnostic potential, we have made systematic studies of ion-gas interactions in the laboratory. Our measurements make use of the Livermore Electron Beam Ion Trap source and of a 36-element microcalorimeter array from the ASTRO-E space flight mission. The charge-exchange-induced $x$-ray spectra provide diagnostic line ratios for measuring the speed of the solar wind, its energy loss, its composition as well as that of the cometary gases. The rich spectral information will make cometary $x$-ray emission an attractive target for satellite observations in the coming years.

\section{Laser-Plasma Interaction Experiments on Nonlinear Saturation of Stimulated Brillouin and Raman Scattering}

Siegfried Glenzer et al.

We are presenting novel Thomson scattering data from plasma waves that nonlinearly scatter the laser light in plasma conditions of general interest to the inertial confinement fusion (ICF) and high energy density science (HEDS) communities. The instabilities of primary concern in the field of laser-plasma interactions are stimulated Raman and Brillouin scattering (SRS and SBS). For ICF, these processes can reflect a large fraction of the incident laser light thereby reducing the efficiency of the target, affecting implosion symmetry, inducing laser damage and causing capsule preheat. For HEDS, large scatter will reduce the achievable radiation temperature and possibly produce non-optimum x-radiation spectra. However, for some applications, large SRS may produce interesting $\mathrm{keV} x$-ray fluxes. The behavior of the plasma waves as measured with Thomson scattering is investigated to establish the viable mechanisms for saturation and to develop the physics basis for target designs in which laser plasma instabilities are included. In support of this experimental initiative, simulations of SRS and SBS from gasbags and hohlraums are performed with realistic models of the laser beam and with saturation models. These models are being developed based on calculations in collaboration with Princeton, LANL, Ecole Polytechnique, LLE, University of Alberta, and UCLA.

\section{Liquid-Bead-Based Biological Detection Assays} Fred Milanovich

The current art of biodiagnostics is inadequate to sufficiently address the broad spectrum ( 30 or more) of biological terrorist threat agents with timely detection and identification or to assay thousands of human exposure victims in a time consistent with successful medical interdiction. Indeed, adequate coverage with today's technology would require the simultaneous deployment of many instruments with varying operating principles or formats, presenting emergency response personnel with a logistical nightmare. We are developing a new technology, the Liquid Array, that has the potential to simultaneously detect the presence of up to 50 
bioterror agents or provide exposure diagnostics to 1000 s of potential victims in a matter of hours. The Liquid Array can detect all threat agent types (bacteria, spores, viruses and toxins) in all potential media (air, water, food, body fluids, etc.).

\section{Making Climate Models Better using Weather Forecasts and Observations}

Gerald Potter et al.

We are using a climate model to predict past weather to see how quickly it drifts from the observed weather and from detailed surface observations measured by the Department of Energy-sponsored Atmospheric Radiation Measurements (ARM) program. If successful, this approach can be used to improve the model components responsible for errors in much the same way that weather prediction centers use short-range forecast verifications to improve their models. Our goal is to improve climate models by making the techniques we develop available to all modeling groups.

\section{Making Peptides in Cometary Impacts}

Jennifer Blank

Using one of the Laboratory's hypervelocity lightgas guns, we're testing organic molecules such as amino acids under shock conditions to see if these building block of life could have survived an impact delivery on Earth via a comet from outer space. Our experimental "comet" is a mixture of liquid water and dissolved amino acids. After placing this mixture in a 1-in. diameter stainless steel canister inside a tank, we fire a metal bullet down a 6.2-m gun barrel into the tank at impact velocities up to $2 \mathrm{~km} / \mathrm{s}$ (about $4500 \mathrm{mph}$ ). The impact of the bullet on the capsule generates pressures of hundreds of thousands of atmospheres, comparable to those experienced by an icy comet striking the Earth at an angle very low to the horizon. Accompanying the impact pressures are high temperatures that many believe sufficient to cause the organic materials to break down. After impact, we extract the liquid inside the canister and analyze it to see how its chemistry has changed. Not only do some of the original amino acids survive, but some also react together to form chains of amino acids-peptides. Our experiments have demonstrated that the energy of an impact can be used to create make larger organic molecules.
Mechanical Properties of Nanocrystalline Nickel-Atomic Scale Simulations and Experimental Observations

Maria J. Caturla

Nanocrystalline metals, with grain sizes below $100 \mathrm{~nm}$, present interesting mechanical properties. As grain size decreases, hardness of these materials increases, following the well-known Hall-Petch relationship. However, breakdown of this relationship is expected at very small grain sizes. We present experimental measurements of nanoindentation hardness in nanocrystalline nickel (Ni), where softening is observed for grain sizes below $\sim 14 \mathrm{~nm}$. This transition is associated with a change in mechanisms of deformation, as revealed by molecular-dynamics simulations for deformation of nanocrystalline $\mathrm{Ni}$ with grain sizes between 4 and $30 \mathrm{~nm}$. At small grain sizes, grain boundary processes such as grain rotation, grain boundary sliding and migration are responsible for deformation. For 12-nm average grain sizes, these simulations show a strong effect of crystallographic texture, with enhanced dislocation activity for low-angle grain boundaries as compared to random grain boundaries. Smaller grain sizes $(4 \mathrm{~nm})$ show no dislocation activity and all the deformation is accommodated at the grain boundaries.

\section{Mechanisms of Pathogenicity}

J. Patrick Fitch and Sandra McCutchen-Maloney

To determine what makes pathogens virulent and capable of overcoming the defenses of a host organism, we are attempting to identify and characterize the genes and proteins involved in virulence and in the response of the host organism. Our initial focus is on Yersinia pestisthe causative agent of plague-because it is a potential bioterrorist threat agent and has an inducible virulence mechanism. Part of the virulence mechanism (Type III secretion) is found in several other animal, plant, and human pathogens that cause very different clinical manifestations. In the past year, we conducted and tested a whole-genome microarray for transcript profiling, demonstrated a rapid method for detecting and identifying transcription factors that effect differential expression, screened protein expression using commercial surface-enhanced laser desorption/ionization mass spectroscopy (SELDI-MS) arrays, investigated new modeling approaches for virulence and tested one on a DNA 
repair pathway, and began host-pathogen

interaction studies and genetic knockout

experiments. Our project legacy will be a national-

security capability for rapidly detecting the

presence of biological threat agents and genetically

modified organisms. This functional

genomics / proteomics platform will be applicable

to other microbes of concern and other avenues of

biological research.

\section{Microfluidic Tools for Biological Sample \\ Preparation}

Robin Miles et al.

The microscale enables unique separation and detection methods. Acoustic nodes are used to concentrate and purify particles, dielectrophoresis (ac electric fields) are used to concentrate and purify particles, a DNA capture chip has micromachined silica structures to concentrate and purify particles, and capillary electrophoresis (dc electric fields) are used to separate and identify DNA by size.

\section{Molecular Dynamics Simulations of the Editing Subunit of E. coli DNA Polymerase III: An Essential Protein in DNA Replication}

Edmund Lau and Felice Lightstone

The process of DNA replication, copying a cell's entire genome, is primarily under the direction of DNA polymerase III (pol III). For E. coli, the multiprotein complex of DNA pol III, consisting of $\sim 10$ different proteins, is able to replicate the genome in $20 \mathrm{~min}$. The core proteins are $\alpha, \varepsilon$, and $\theta$, where $\alpha$ is the polymerase, $\varepsilon$ is the editing enzyme, and $\theta$ has unknown function. The $\varepsilon$ subunit helps maintain the high fidelity of pol III. This enzyme has recently been crystallized with $5^{\prime}$ p-nitrophenyl ester in the active site. We have used this crystal structure as a starting conformation for a classical molecular-dynamics study. A single stranded trinucleotide substrate was modeled into the active site of $\varepsilon$. Three classical molecular dynamics simulations were performed where His162 is in three different protonation states. An additional simulation with the active site containing the gas-phase transition state was also performed.

\section{Nanoscience and Nanotechnology for Nonproliferation Applications}

John Reynolds and Thomas Tillotson

In this project, we are developing new nanostructured and nanoporous materials that are highly selective and specific for chemicals indicative of the production of chemical and biological weapons. We synthesized different organic-inorganic sol-gels with silica, oxidizing the organic substituent to prepare nanostructured materials with bimodal pore size distributions. We also demonstrated that carbon nanotubes can be used as thin-film coatings. These nanotube coatings have been favorably compared to stateof-the-art carboxen thin-film coatings and provide an alternative coating in the event carboxen 1066 becomes unavailable or too costly. This project is cofunded with the Chemistry and Materials Science Directorate and the Laboratory Directed Research and Development Program

\section{The National Ignition Facility}

Regina Bonanno

The National Ignition Facility (NIF) will be a national facility for high-energy-density and inertial-fusion-energy science in support of stockpile stewardship, basic science, and fusionenergy studies. NIF is a stadium-sized 192-beam laser system that will provide up to $1.8 \mathrm{MJ}$ of ultraviolet laser energy to targets located in its 10$\mathrm{m}$-diameter target chamber. NIF is scheduled to be completed in 2008, and first lightcommissioning of 4 laser beams-will be achieved in the coming year. Soon after first light, experiments are planned to begin studying the physics of materials subjected to conditions of extreme temperature and pressure. Other experiments will study laser-plasma interactions and turbulent hydrodynamics. By the time NIF is completed, over 1000 experiments will have been performed. After completion NIF will provide experimenters with over 700 shots per year along with a core set of $\mathrm{x}$-ray and neutron diagnostics and data-acquisition systems. NIF's ultimate goal is the achievement of fusion ignition and burn with energy gain. NIF's indirect drive configuration will be used to focus laser beams into a hohlraum surrounding a small deuterium-tritium capsule where $x$ rays will ablate the surface of the capsule and cause the fusion fuel to compress and ignite the fusion process. 


\section{Neutron Detectors for Inertial Confinement Fusion}

Michael Moran et al.

Most of the energy produced by inertial confinement fusion (ICF) is released in the form of neutrons traveling with very high velocities. Measurements of these energetic neutrons provide detailed information about physical conditions at the instant of fusion in ICF experiments. Simple materials can be used to assemble a low-intensity neutron source, and detectors can measure the resulting neutron fluxes. Surprisingly similar measurements in ICF experiments can study the temperatures of the nuclear-fuel "burn," record the time history of the nuclear reactions, and record a neutron picture of the nuclear source. These kinds of measurements will be important in the effort to design experiments that achieve nuclear ignition at the National Ignition Facility.

\section{OPCW Certification for the Forensic Science Center}

Armando Alcaraz

The mission of the Organization for the Prohibition of Chemical Weapons (OPCW) is to implement the provisions of the Chemical Weapons Convention. The OPCW envisions a world free of chemical weapons and cooperation in chemistry for peaceful purposes among all nations. To accomplish this goal, the OPCW proposes policies for implementation of the Convention. When the United States signed this treaty in 1997, it agreed to support the OPCW inspection programs for verifying the destruction of chemical weapons and helping prevent their reemergence while simultaneously protecting legitimate national security and commercial business interests. The OPCW requires two accredited laboratories to independently analyze the samples; a Congressional mandate requires that no sample leave the U.S. for analysis during an OPCW inspection. LLNL was recently selected to become the second accredited analytical laboratory in the U.S. The poster presents an overview of the process for achieving accreditation, our current status, and the nature of the staff and facilities needed to meet the demanding requirements.
Precision Engineering-Advanced Manufacturing for the NIF Final Optics

Keith Carlisle, Paul Geraghty, and Sam Thompson

This poster will outline the key developments in precision engineering design and manufacturing technology that enabled LLNL to fabricate largeaperture optics capable of meeting the demanding operational requirements for the National Ignition Facility (NIF) final optics assembly. In particular, it will describe the engineering achievements in machine design that made it possible to fabricate large-aperture $\left(42 \mathrm{~cm}^{2}\right)$ potassium dihydrogen phosphorous (KDP) harmonic generators to achieve a required surface roughness over the full aperture to less than 2-nm rms with no surface damage. Details of how crystal alignment or phase-match angle for frequency conversion could be held to be better than $10 \mathrm{mrad}$, including a transmitted wavefront gradient or nonlinear thickness variation to better than $11 \mathrm{~nm} / \mathrm{cm} \mathrm{rms}$.

Meeting these difficult requirements required an elaborate fabrication process. This poster describes the fabrication process starting from a single crystal grown from a seed, to the final finished KDP optic requiring over 60 manufacturing operations from sawing, milling, diamond flycutting, to finishing and characterization. Details of the critical design features for the Finishing Machine (a single-point-diamond fly-cutting machine) will be described, including how a 100$\mathrm{kg}$ aluminum fly cutter can be spun at $1000 \mathrm{rpm}$ with a maximum error motion of less than $25 \mathrm{~nm}$ and asynchronous error motion below $12 \mathrm{~nm}$; why its speed had to be controlled to better than $0.01 \%$ to maintain crystal flatness; why temperature control to $0.01^{\circ} \mathrm{C}$ of the $5000-\mathrm{kg}$ machine structure was necessary for nanometer position control; and why the single-crystal-diamond tool geometry was so critical to surface roughness and reduction of surface damage.

\section{Quantum-Level Calculations}

Giulia Galli et al.

As we enter a new century, growth in computer power and the coming of age of first-principles theories have positioned physicists to address anew the complexity of matter at the microscopic level, treating each atomic constituent in a detailed manner. Using Advanced Simulation and Computing (ASCI) supercomputers, along with optimized codes and algorithms, the properties of 
matter can be obtained from the fundamental laws of quantum mechanics in a fully predictive way, without input from experiments. We give examples of the deep insight into high-pressure physics and the new exciting area of nanoscience that can be gained using quantum simulations. In particular, we present results on the dynamic activity of atoms and molecules in compressed, hot fluids, e.g. hydrogen and water, and results on nanomaterials, including both organic and inorganic nanoparticles. At the nanoscale, matter behaves in a complex way that is both different from the behavior of bulk systems and, in general, difficult to measure. Quantum simulations are ideal tools to study matter at the nanoscale and explore its potential applications for new devices.

\section{Remote Optical Characterization Sensor Suite} Joel Bowers

We are developing remote-sensing instruments and spectroscopic analysis for ballistic missile defense applications. Our ultimate goal is to develop technology for real-time characterization of impact and debris to provide battlefield commanders with rapid identification of enemy warheads carrying weapons of mass destruction. We are looking at the optical signatures that might be accessible to remote-sensing instruments following the intercept of a hostile missile. Exploitation of remotely detected optical signatures requires knowledge of threedimensional breakup dynamics, atmospheric chemistry and transmission, as well as materials spectroscopy to determine appropriate signatures and remote sensing opportunities. The integrated system we've developed, called the Remote Optical Characterization Sensor Suite (ROCSS), incorporates infrared and visible spectrometers, radiometers, and fast-framing digital video. The sensor suite has been flown on a Gulfstream II aircraft called the High Altitude Observatory (HALO) operated for the U.S. Army Space and Missile Defense Command. ROCSS successfully collected airborne sensor data from a live-fire intercept test at the Reagan Test Site.

\section{Removing Nitrate and Perchlorate from Groundwater using the Containerized Wetland System}

Paula Krauter and William Daily

Remediation strategies for the removal of nitrate and perchlorate based on adsorption by activated carbon are not effective due to rapid saturation of adsorption sites, and competition with other anions such as nitrate. Other remediation processes include reverse osmosis and ion exchange; however, both are relatively expensive and produce hazardous waste of salts and perchlorate. A new remediation process for nitrate- and perchlorate-contaminated groundwater has been under evaluation at Site 300 since November 2000. This remediation process is groundwater flow through a wetland bioreactor. The bioreactor was first started on November 13, 2000. During its time of operation, it successfully reduced influent nitrate concentrations from about $46,000 \mu \mathrm{g} / 1$ to levels below the detection limit of $500 \mu \mathrm{g} / 1$, and perchlorate from $14 \mu \mathrm{g} / 1$ to below the detection limit of $4 \mu \mathrm{g} / 1$.

\section{Ribbon Fiber Lasers-the High-Power Lasers of the Future}

Jay Dawson, Zhi Liao, Raymond Beach, and Steven Payne

Optical fiber lasers are diode-pumped solid-state lasers in which the gain media and many of the laser components operate based on waveguide physics rather than conventional diffraction- and refraction-based bulk optics. These lasers are typically compact, robust turnkey devices with high efficiency, high beam quality and high reliability. Historically, the power output of fiber lasers has been too low to be of interest in most Department of Energy (DOE) applications.

However, output powers and pulse energies from fiber lasers have been increasing by a factor of 10 every three years since 1985. Recently, average output power from both pulsed and CW fiber laser has exceeded $100 \mathrm{~W}$. Thus these devices are now entering realms of interest to DOE applications. The poster presents a vision for future LLNL leadership based on the ribbon fiber laser in the exciting field of high-power fiber lasers. 
A Search for the Origin of Matter/Antimatter Asymmetry: The BABAR Experiment at the SLAC-LBNL-LLNL B Factory

Douglas Wright et al.

The existence of charge/ parity $(\mathrm{CP})$ violation, a fundamental process that favors particles over antiparticles, remains a crucial problem for both particle physics and cosmology. $\mathrm{CP}$ violation is a necessary ingredient in cosmological models that result in a universe dominated by matter over antimatter, such as the universe in which we live. Only one $\mathrm{CP}$-violating process has ever been discovered, but the observed effect, within the context of current particle theory, is too small to provide the mechanism required by cosmology. Either the current theory is incomplete, or the $\mathrm{CP}$ violation required by cosmology has remained undetected. An international collaboration of particle physicists has assembled an accelerator and detector facility at Stanford to address the mystery of $\mathrm{CP}$ violation. The experiment studies the decay of $B$ mesons, a heavy and rare form of nuclear matter. By measuring the asymmetry between the decays of $B$ and anti-B mesons we will, for the first time, be able to confront the standard theory predictions about $\mathrm{CP}$ violation.

\section{Secure Air-Optic Transport and Routing Network}

Anthony Ruggiero

Information collection and communication technologies are crucial to our national security. While new generations of U.S. sensor systems insure our technological edge by enabling unprecedented quantities of data to be collected, communication bottlenecks often limit its timely synthesis and interpretation. To maintain our information dominance in the next century, secure high-capacity communication networks will be essential. They will require global coverage with seamless routing of data between permanent infrastructure, such as the fiber internet, and deployable infrastructure that includes mobile and temporary platforms. Open-air laser communication (lasercom) links are a key enabling technology for fulfilling this vision. At LLNL, we are building a secure air-optic transport and routing network (SATRN) to develop and demonstrate key enabling technologies for longrange, secure, high-capacity, high-availability, airoptic laser communications links. Through a series of system-level horizontal-path link experiments, we are establishing the viability of our technology in transporting high-capacity sensor data over long ranges. Air-optic link data from our horizontal lasercom demonstration testbeds can be used to calibrate performance models and simulations of terrestrial links to airborne intelligence, surveillance, and reconnaissance platforms.

\section{A Simulations and Experimental Program to Assess Ballistic Missile Defense Lethality Douglas Faux et al.}

LLNL has been involved in assessing missile system effectiveness against nuclear threats for the past 25 years. Our unique knowledge of nuclear weapons enables us to evaluate the damage and its effect on nuclear warheads. The Ground-Based Midcourse Defense (GMD) Program, sponsored by the Missile Defense Agency (MDA) was established to help protect the U.S. from ballistic missile attacks. To support the GMD Lethality program, LLNL provides kinetic-energy lethality criteria for weapons of mass destruction. To accomplish this task we evaluate and design nuclear threats, fabricate test targets, support hypervelocity impact experiments and perform detailed numerical simulation to assess the lethality of the intercept. These activities are all necessary to provide MDA the needed kill criteria to assess the effectiveness of the GMD system.

\section{Solid-Oxide Fuel Cell Development at LLNL Quoc Pham et al.}

We are developing advanced solid-oxide fuel-cell (SOFC) technology for stationary and mobile applications. SOFCs are solid-state electrochemical devices that produce electricity directly from chemical fuels. SOFCs have very low toxic emissions (hydrocarbons, carbon monoxide, nitrogen oxide, sulfur compounds, etc.) because there is no direct combustion of the fuels. Due to their higher efficiency, SOFCs also have lower greenhouse gas emissions than conventional power-generation devices. The SOFC development program at LLNL began in 1998 as a Laboratory Directed Research and Development project. Since then, the program has attracted several external funding sources. Major accomplishments include the development of a low-cost fabrication, thin-film-deposition 
technique; the development of a high-

performance electrode; and the demonstration of the world's highest power density SOFC stack.

We are now in the process of scaling up the effort to demonstrate a 1-kW SOFC stack operating on natural gas.

\section{Solid-State Heat Capacity Lasers, a Compact Missile-Defense System \\ Mark Rotter}

Under the support of the U.S. Army's Space and Missile Defense Command, and in collaboration with industrial partners (General Atomics, Northrup/Grumman Poly-Scientific, Decade Optical Systems, and others), we are developing high-average-power (100-kW-class) heat-capacity laser technology for applications in tactical shortrange air defense missions. The ultimate vision is an electrically powered, solid-state weapon that can be deployed on a hybrid electric vehicle. To establish a solid technical basis for the heatcapacity laser operation, we have built a $10-\mathrm{kW}$ prototype (9-disk Nd:glass laser pumped by flashlamps). This laser was delivered to the Army's High Energy Laser Systems Test Facility (HELSTF) at White Sands Missile Range during the summer of 2001. On August 31, 2001 our milestone of $10-\mathrm{kW}$ average power for $10 \mathrm{~s}$ was exceeded when we obtained an average energy / pulse of $640 \mathrm{~J}$. With the $20-\mathrm{Hz}$ repetition rate, this resulted in an average power approaching $13 \mathrm{~kW}$ during the 10-s burst. Future plans call for a 100-kW-class, diode-pumped, solid-state laser utilizing crystalline laser media for better thermal characteristics and operating efficiency. We are currently building a small-scale prototype of such a laser to be activated this year.

\section{The Spheromak Path to Economic Fusion Energy}

Simon Woodruff

Most paths to magnetic fusion energy entail trapping hot plasma in magnetic bottles with external field coils, leading to rather complicated and expensive devices. The spheromak is markedly different in that the confining fields are generated by the currents in the plasma, and the most favorable configuration is found naturally by "relaxation" to a nearly spherical minimumenergy state. Furthermore, the heating and current drive processes are intrinsic, and so the spheromak offers potentially the simplest (and hence most attractive) path to economic fusion energy. Possibly obstructing this path are confinement and field-generation issues-we need to show that we can sustain a spheromak with reasonable confinement, and show that we understand the mechanisms that generate the field (and drive current). In our poster, we highlight the main features of the spheromak and present some recent results to show how we are addressing confinement and field generation.

\section{Stretchable Micro-Electrode Array}

Peter Krulevitch and Mariam Maghribi

Millions of people suffering from diseases such as retinitis pigmentosa and macular degeneration are legally blind due to the loss of photoreceptor function; however the neural cells to which the photoreceptors normally synapse remain viable, and electrical stimulation of these cells has been shown to result in visual perception. These findings have generated worldwide efforts to develop a retinal prosthesis device, with the hope of restoring vision to millions of patients. With the advancement of microfabrication, integrated circuits, and wireless technologies, the once inconceivable goal of developing such a system now appears to be within reach. As part of a multilaboratory research team to create a retinal prosthesis, we are developing an implantable, stretchable micro-electrode array using polymerbased microfabrication techniques. The device will serve as the interface between an electronic imaging system and the human eye, directly stimulating retinal neurons via thin-film conducting traces and electroplated electrodes.

Because the device is intended as a long-term implant that interfaces with delicate retinal cells, vital biological and physical design requirements must be met. The metal features are embedded within a thin substrate fabricated using poly (dimethylsiloxane) (PDMS), a biocompatible elastomeric material that is impermeable to water. The conformable nature of PDMS is critical for ensuring uniform contact with the curved surface of the retina. Robustness of the micro-electrode array is another important design consideration, as stretching and bending occur during fabrication and implantation of the device. 
Surface Passivation Effects of Deposited Germanium Nanocrystal Films Probed with Synchrotron Radiation

Christoph Bostedt et al.

Nanocrystals and clusters represent a new class of materials that exhibit promising novel electronic, catalytic, or magnetic properties. We are investigating their special electronic properties as a function of size and surface passivation and exploring the possibility of building new materials with these nanostructures. In our experiments, the nanocrystals are condensed out of the gas phase and subsequently deposited on various substrates. Changes of the electronic structure as a function of nancocrystal size and surface passivation are measured using synchrotron-radiation-based spectroscopy techniques. With this work we can answer basic questions concerning the structure-property relationship of clusters and nanocrystals. Our work is an experimental demonstration of quantum confinement theory and one of the first studies aimed at understanding surface passivation effects in nanoclusters.

\section{Thermodynamics and Structure of Plutonium Alloys}

Patrick Allen et al.

Plutonium $(\mathrm{Pu})$ is perhaps the most complex element in the periodic table, displaying a wide array of unusual chemical, structural, and metallurgical properties. For example, Pu may adopt one of six different structures $\left(\alpha, \beta, \gamma, \delta, \delta^{\prime}\right.$, and $\varepsilon$ ) upon heating from room temperature to its melting point of $913 \mathrm{~K}$. Furthermore, the addition of alloying elements to $\mathrm{Pu}$ has dramatic effects on its phase behavior, presenting numerous fundamental research challenges. Our research effort seeks to understand the role of alloying elements on $\mathrm{Pu}$ phase behavior from both a theoretical and an experimental perspective. We are using semi-empirical predictive tools combined with basic metallurgical synthesis and characterization methods to enhance our understanding of the thermodynamics (energetics, phase diagrams) and structure (crystallography, microstructure) in Pu alloy systems.
The True Heart of a Star

David Dearborn

Stars are much more than faint objects twinkling in the night sky. They provide the fundamental quantitative measures for the universe, setting the scale(s) for distances, and limits on the age. Stars are also used as physics laboratories, constraining atomic, nuclear, and particle properties and providing observational signatures that challenges our understanding of complex physical processes. Current practices in stellar evolution employ one-dimensional calculations that apply only to a minority of the observed stars (single nonrotating stars, or welldetached binaries). Even in these systems, astrophysicists are dependent on models of complex three dimensional (3-D) processes like convection. With the advent of massively parallel computers and the development of codes to use them, LLNL was uniquely situated to begin understanding stars without this artificial limit. Early results of modeling whole stars in 3-D will be presented.

\section{Understanding Global Ozone and Aerosol Pollution using the LLNL IMPACT Chemistry Model}

Douglas Rotman et al.

We present the LLNL Integrated Massively Parallel Atmospheric Chemical Transport (IMPACT) model. This model simulates chemical species in the troposphere (lower atmosphere) and stratosphere (upper atmosphere), with a focus on ozone and the processes that affect its distribution. Ozone is important (and helpful) in the stratosphere where it absorbs harmful UVB radiation and is important (and destructive) in the troposphere where it causes respiratory problems and harms materials and agricultural crops. The model can also simulate the distribution of aerosols-tiny particles of carbon, sulfur, and dust - that cause local air pollution and globally affect climate. We use the IMPACT model to examine how energy use emissions, including aerosols, carbon monoxide and nitrogen oxides, affect global ozone and how winds can transport ozone, dust, and other aerosols great distances to influence local air pollution. 
Using Human Urinary PhIP Metabolites to Study Individual Variation in Carcinogen Metabolism and Chemoprevention Through Dietary Interactions

Kristen Kulp, Mark Knize, and James Felton

The metabolism of a chemical that is commonly formed in well-done cooked meat may have an impact on human cancer risk. The genotoxic heterocyclic amine 2-amino-1-methyl-6phenylimidazo [4,5-b]-pyridine (PhIP) is a naturally occurring mutagen that is formed in well-cooked muscle meats. PhIP consistently causes dosedependent breast and prostate tumor formation in rats, and consumption of well-done meat has been linked to increased risk of breast cancer in women. To investigate human metabolism of PhIP at dietary doses we devised an assay to quantify PhIP metabolites in human urine following a single meal of well-done chicken. Although developed to study variation in PhIP metabolism in individuals, our method can also be used to determine the interaction of PhIP with other foods that have been identified as protective for cancer. This newly developed methodology will allow us to assess potential prevention strategies that reduce the possible risks associated with PhIP exposure.

\section{Views of the Solar System using Adaptive Optics at the Keck Observatory \\ Seran Gibbard}

Using the W.M. Keck Observatory adaptive optics (AO) system, we are imaging Solar System bodies at a spatial resolution that is at least 10 times better than conventional imaging without AO. This produces very-high-spatial-resolution images of planetary bodies that allow us to study the details of weather in the atmospheres of Uranus and Neptune, to image in great detail the rings and satellites of Uranus, and to examine the surface and atmosphere of Saturn's large moon, Titan. Neptune and Uranus have atmospheric features that change on short timescales; we are investigating their altitude, composition, and connection to events occurring deeper in the planets' tropospheres. Titan has a solid surface located under its atmosphere, the composition of which is still quite uncertain. To add to our knowledge of Titan's surface, we are determining its surface reflectivity at near-infrared wavelengths and studying the vertical structure of Titan's atmosphere. Preliminary images of Uranus obtained in December 2001 show that AO can resolve individual rings (not possible even with the Hubble Space Telescope, and indicate that it may be possible to observe "ring shepherd" moons that have not been previously detected.

\section{What's New in University Relations Physical Biosciences Institute UC Merced}

Laura Gilliom

The mission of the University Relations Program (URP) is to foster collaborations between LLNL and academic institutions, principally the University of California (UC), that enhance science and technology vitality, and recruiting and mission execution. URP's Science Day posters highlight recent program developments designed to promote these collaborations and two special initiatives.

As of October 1, 2002, an LLNL institute in Physical Biosciences will be established. In addition, URP is serving as a focal point for the Laboratory's active assistance in establishing UC's tenth campus at Merced, which is scheduled to open in the fall of 2004.

URP is increasingly focused on enhancing the Laboratory's recruiting pipeline. We coordinate the Lawrence Livermore Postdoctoral Fellows Program and have recently implemented the LLNL Sabbatical Program to encourage more visiting faculty. URP has also implemented a new effort known as the University Outreach Initiative, which has targeted four university campuses (UCLA, UCSD, the University of Arizona and the University of Illinois at Urbana/Champaign) with a focus on developing new research collaborations and opening new channels for recruiting students and postdocs as future Laboratory employees. UCSB will soon be added to this initiative. URP has also helped facilitate strategic university collaborations with the UC Davis Cancer Center, the Center for Adaptive Optics at UC Santa Cruz and the new Center for Biophotonics at UC Davis. LLNL is now an integral part of two of the eleven National Science Foundation Science and Technology Centers. 
The World's Largest Linux Cluster for LLNL Scientific Simulations

Lynn Kissel and Mark Seager

With the advent of commodity computing platforms, we are now building highly scalable compute engines for scientific simulations. MCR, the worlds largest Linux cluster at 11.1 TFLOPs peak, will bring unprecedented computing capability to LLNL scientists and engineers to solve large and complex physical unclassified scientific problems. Over 26 different applications areas are currently targeted to utilize MCR to meet their demanding programmatic deliverables. MCR will enable world-class computational science in many areas: from biology to materials, from the National Ignition Facility (NIF) to the National Atmospheric Release Advisory Center (NARAC). MCR is currently being built through partnership with Linux NetworX, Quadrics, BlueArc and Cluster File Systems, Inc., and is targeted to have 1,152 dual Pentium 4 nodes with 4.61 TB of memory, 138 TB of local disk and 100 TB of global disk. Target delivery for MCR is in early October 2002. Initial science runs will start in November 2002. 